Rev. Adv. Mater. Sci. 56 (2018) 1-20

\title{
A REVIEW ON MECHANICAL AND TRIBOLOGICAL PROPERTIES OF GRAPHITE REINFORCED SELF LUBRICATING HYBRID METAL MATRIX COMPOSITES
}

\author{
S.K. Khatkar, N.M. Suri, S. Kant and Pankaj \\ Department of Production and Industrial Engineering, PEC University of Technology, Chandigarh-160012, India
}

Received: October 28, 2017

\begin{abstract}
Hybrid metal matrix composites (HMMC's) are next generation metal matrix composite materials that have the potential of gratifying recent demands of advanced energy applications in the field of aerospace, automobile and biomedical. The MMC's have to work at elevated temperature or vacuum environment where lubrication using liquid lubricants is ineffective or difficult to achieve; moreover, liquid lubricants are not environmental friendly, since they releases pollutants into environment. Solid lubricants such as graphite, CNT's, MoS 2 and graphene have been widely used as secondary reinforcements to meet such operational needs by fabricating green or environmental friendly self lubricating HMMC's. This article outlines recent advances in the area of self lubricating HMMC's reinforced with graphite as secondary reinforcement. The focus of the study was on mechanical and tribological behavior of Aluminum and magnesium based self lubricating HMMC's. The effect of graphite, different hybrid reinforcements and their strengthening mechanisms are also discussed in detail.
\end{abstract}

\section{INTRODUCTION}

Current engineering applications demand stronger, lighter and less expensive materials. A good example is the current interest in development of new structural materials for transportation industry that have higher strength to weight ratio which can reduce overall the fuel consumption, weight and green house emissions. In recent years much attention has been paid to light weight aluminum and magnesium composite materials with broader spectrum of properties which is impossible to achieve using monolithic materials [1]. The important properties of these light weight composites include: low density, high specific modulus, thermal conductivity, strength, wear resistance, good fatigue response and superior stability at elevated temperature [1-5].

Metal matrix composites (MMC's) are an important class of advance engineering materials that are fast replacing conventional metallic alloys not only in aerospace and automobile industries but also in defense, marine, sports and recreation industries due to their excellent combination of properties[6]. The MMC's combine the properties such as ductility and toughness of metallic alloys with high strength, modulus and temperature resistance of reinforcement materials to achieve desirable properties like wear resistance, high strength, light weight, self cleaning, self lubricating, high thermal conductivity and low cost for automobile applications as illustrated in Table 1. The common metallic alloys utilized for fabrication of MMC's are alloys of light metals such as Aluminum, magnesium and titanium; however another metallic alloys such as nickel, copper and stainless steel has been extensively used. Aluminum and magnesium alloys remain the most utilized metallic alloys as matrix

Corresponding author: S.K. Khatkar, e-mail: sandeepkhatkar99@gmail.com 
Table1. MMCs being developed for use in automotive applications, adapted from [27].

\begin{tabular}{|c|c|c|}
\hline Properties & Materials & Applications \\
\hline Wear resistance & $\begin{array}{l}\mathrm{SiC}, \mathrm{Al}_{2} \mathrm{O}_{3} \text {, and/or graphite } \\
\text { reinforced micro and nano MMCs }\end{array}$ & $\begin{array}{l}\text { Bearing surfaces, liners, pistons, cam } \\
\text { shafts, tappets, lifters, rockers, brake } \\
\text { components }\end{array}$ \\
\hline $\begin{array}{l}\text { Light weight, } \\
\text { energy absorption }\end{array}$ & $\begin{array}{l}\text { Fly ash cenosphere- and low-density } \\
\text { ceramic microballoon-reinforced } \\
\text { syntactic foam MMCs }\end{array}$ & $\begin{array}{l}\text { Crumple zones, frame members and } \\
\text { reinforcements, pedestrian impact } \\
\text { zones, batteries }\end{array}$ \\
\hline Self-cleaning & $\begin{array}{l}\text { MMCs with hydrophobic } \\
\text { reinforcements }\end{array}$ & $\begin{array}{l}\text { Biomimetic coatings, and surface } \\
\text { finishes Water pumps, water jackets, } \\
\text { exposed metallic components }\end{array}$ \\
\hline Self-lubricating & $\begin{array}{l}\text { Micro and nano MMCs } \\
\text { incorporating graphite, } \mathrm{MoS}_{2}, \mathrm{TiB}_{2} \text {, } \\
\text { hexagonal BN, or other } \\
\text { solid lubricants }\end{array}$ & $\begin{array}{l}\text { Bearing journals, cylinder liners, pistons, } \\
\text { cv joints, gear surfaces }\end{array}$ \\
\hline $\begin{array}{l}\text { High thermal } \\
\text { conductivity }\end{array}$ & $\begin{array}{l}\text { Micro and nano MMCs reinforced } \\
\text { with high-conductivity carbon, } \\
\text { diamond, or cubic boron nitride } \\
\text { (CBN) powder }\end{array}$ & $\begin{array}{l}\text { Cylinder liners, water passages, brake } \\
\text { components, turbo/supercharger } \\
\text { components, catalytic converters, } \\
\text { electronics packaging }\end{array}$ \\
\hline High strength & $\begin{array}{l}\text { Micro and nano MMCs reinforced } \\
\text { with } \mathrm{SiC} \text { or } \mathrm{Al}_{2} \mathrm{O}_{3} \text { particles, CNTs, } \\
\text { carbon or Nextel fibers, and in } \\
\text { situ ceramics }\end{array}$ & $\begin{array}{l}\text { Connecting rods, brake calipers, brake } \\
\text { rotors, brake calipers }\end{array}$ \\
\hline Low cost & $\begin{array}{l}\text { MMCs containing fly ash or waste } \\
\text { sand as fillers }\end{array}$ & $\begin{array}{l}\text { Intake manifolds, accessory brackets, } \\
\text { low-load brackets, oil pans, valve covers, } \\
\text { alternator covers, water pumps }\end{array}$ \\
\hline
\end{tabular}

material both in research and industrial view point [1]. Low density and high strength to weight ratio of aluminum and magnesium make both of them a potential candidate for advanced light weight applications [1]. Aluminum and magnesium based MMC's can reduce the overall weight, fuel consumption and green house emissions in automobiles and aircrafts [1,2]. Every $10 \%$ of weight reduction from the average car or light truck can lead to reduction of fuel consumption by $6.9 \%$ [1] and as stated by W.A. Manteiro et al. [7] the reduction of $100 \mathrm{kgs}$ weight in net load reduces fuel consumption by half liter per 100 kms. It causes 2000 kg less $\mathrm{CO}_{2}$ production during the mean life of an automobile.

The tremendous benefits of improved fuel efficiency with less pollutant emission put a strong demand of light weight materials with excellent mechanical and tribological properties. Satisfying the demand of superior mechanical and tribological properties aluminum and magnesium composite and hybrid composite materials are fabricated by reinforcing different reinforcement materials such as $\mathrm{SiC}$ [8-12], $\mathrm{Al}_{2} \mathrm{O}_{3}$ [13-16], TiC [17], $\mathrm{B}_{4} \mathrm{C}[15,18,19], \mathrm{CeF}_{3}$
[20], Graphite [8,21-24], CNT [25], graphene nanoplatelets [26], and $\mathrm{MOS}_{2}$ [27] for the light weight application.

During these applications, MMC's component has to work in extreme temperature and vacuum environment during sliding where lubrication by liquid lubricant is ineffective or difficult; moreover liquid lubricants are not environmental friendly, releases pollutants into environment. In such circumstances, the materials enable to sustain low coefficient of friction and wear rate without providing external lubrication can be used, these are known as self lubricating materials [28]. The demand of self

lubricating materials reinforced with MMC's is increasing for environment-friendly or green tribology, sustainability and energy efficiency. According to P.K. Rohatangi et al. [28], self lubricating materials can be fabricated by two methods as below:

i) Through applying coating films to materials such as Diamond like carbon (DLC)film [29-33] and boron nitride.

ii) Through reinforcing solid lubricants such as graphite [11-15,18,34-48], MoS $_{2}[49,50]$, CNT [51-53] and 


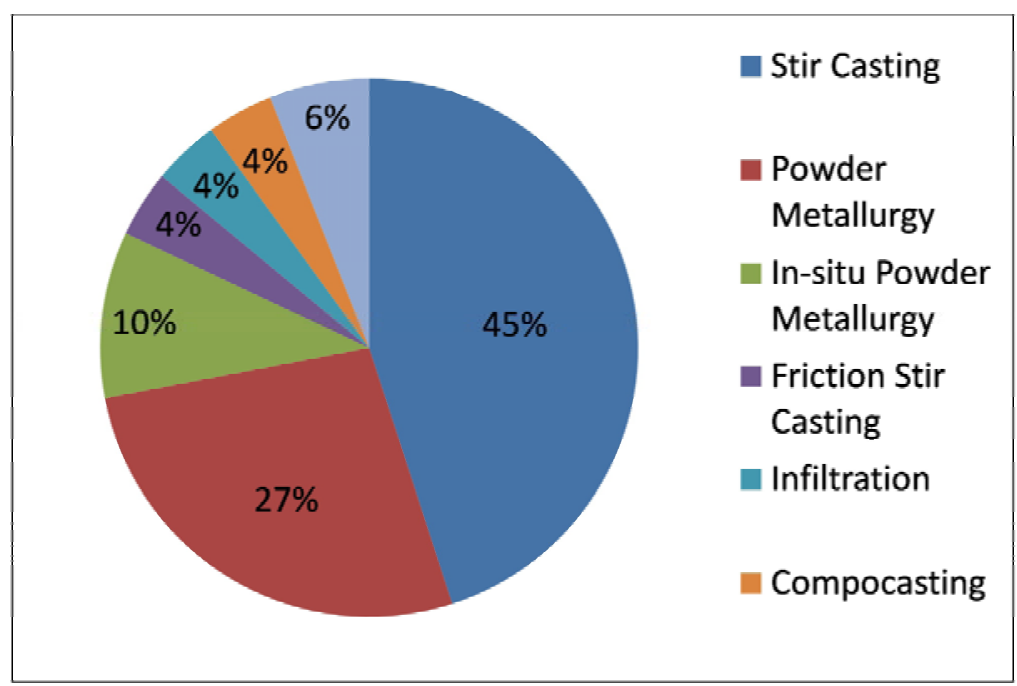

Fig. 1. Contribution (\%) of different fabrication techniques for $\mathrm{Al}$ and $\mathrm{Mg}$ based MMHCs reviewed in this report.

Graphene $[26,54,55]$ into matrix of composite materials.

The main emphasis is given to second method of fabrication of self lubricating MMC's through embedding solid lubricants since they posses higher fatigue life, superior corrosion resistance and tremendous tribological properties than the former coating method [28].

With an increasing reinforcing percentage of solid lubricant such as graphite in matrix material, the wear rate decreases due to formation of tribo-layer of solid lubricant between sliding contact area; however hardness of self lubricating MMC's decreases due to presence of soft solid material $[8,24,34,56$ 61]. In another point of view adding denser reinforcement such as $\mathrm{SiC}, \mathrm{B}_{4} \mathrm{C}$, and $\mathrm{Al}_{2} \mathrm{O}_{3}$, increases the weight of MMC's, depending on reinforcement content $[12,18,57]$. Reinforcing these harder particles into matrix increases hardness of MMC's $[10,11,38]$ resulting difficulty in machining of MMC's. Along with this problem the high cost of ceramics and limited supply of ceramics reinforcing materials in developing countries motivates the formation of composite materials by using multiple reinforcement, known as hybrid metal matrix composite (HMMC). Self lubricating hybrid metal matrix composites are those where hard ceramic materials with excellent strength are used as primary reinforcement and solid lubricants such as graphite,MoS ${ }_{2}, \mathrm{CNT}$, and Graphene with lower cost, lower density and having self lubricating properties are used as secondary reinforcement to achieve better mechanical and tribological properties. Graphite reinforced Al and Mg based HMMC's has been successfully fabricated through stir casting, powder metallurgy, infiltration method, compo casting, friction stir processing, liquid metallurgy, stir casting hybridized with squeeze casting and in-situ powder metallurgy. The percentage contribution of these different techniques used by earlier researchers is illustrated in Fig. 1. Stir casting $(45 \%)$ was reported as the most contributing technique followed by powder metallurgy $(27 \%)$ for graphite impregnated self lubricating hybrid composites of $\mathrm{Al}$ and $\mathrm{Mg}$. The most contribution of this liquid state processing is due to its commercial viability, flexibility, simplicity, cost effectiveness, wider ranges of complex shapes and less damage to reinforcement particulates. However inhomogeneous distribution of reinforcement, porosity, poor wettability, clustering, segregation and interfacial reaction are the matter of concern about stir casting $[18,19,40]$. Powder metallurgy is an important solid state processing technique having following tremendous advantages i) Nano sized reinforcements can be uniformly distributed. ii) Avoid particle clustering, agglomeration, wettability and formation of unwanted secondary phases which is common during liquid state processing.iii) hybrid composites with higher reinforcement's content can be successfully fabricated $[10,39,43,46]$.

In this article the ability of graphite as a solid lubricant has been conceptualized. The mechanical and tribological properties has been studied of self lubricating HMMC's reinforced with ceramics particles like $\mathrm{SIC}, \mathrm{Al}_{2} \mathrm{O}_{3}, \mathrm{TiC}, \mathrm{B}_{4} \mathrm{C}$ as primary reinforcement and graphite as secondary reinforcement. The main aim of this review is to give an overview on current progress of research in area of self lubricating HMMC's reinforced with graphite as secondary reinforcement. The focus is on mechanical and 
tribological behavior of aluminum and magnesium based self lubricating HMMC's and effect of graphite on these properties has also been described.

\section{GRAPHITE A SOLID LUBRICANT}

Graphite is one of the three allotropic forms of element carbon, structurally composed of carbon layer lattice lamella crystal structure that is hexagonal in orientation. The covalent bond between the rings of carbon atoms in crystal structure is stronger than vanderwaal bond between layers. These Van der Waals forces break during sliding force, resulting a low coefficient of friction ( $\mu=0.1$ to 0.2$)$ for graphite while sliding on a clean surface, however providing good loading strength vertically [28]. The broad applications of graphite in many industries are due to its low cost, excellent in-plane mechanical, structural, thermal and electrical properties as illustrated in Table 2. Graphite also shows great potential as a lubricant in different forms such as a thin film, colloidal dispersion, bulk, powder, solid and composite forms to counter frictional force and wear drag [62]. Graphite whether natural or synthetic, its powder with average size of $4-5 \mu \mathrm{m}$ produced good lubrication effects and can reduce to a minimum friction and wear of specimen [63].

To achieve excellent tribological properties continuous flow of lubricant between two sliding surfaces is very important. This is easily maintained when fluid lubricants are used. In order to insure the continuous flow in case of solid lubricant, an excellent innovation is to reinforce solid lubricant (Graphite and $\mathrm{MoS}_{2}$ ) into matrix of sliding components by fabricating a metal matrix composite (i.e. when solid lubricant is added as primary reinforcement) and hybrid matrix composites (i.e. when solid lubricant is added as secondary reinforcement).

\section{GRAPHITE REINFORCED HYBRID COMPOSITE}

Aluminum and magnesium alloys having application in automobile illustrate different types of wear mechanism during sliding motion of two surfaces includes abrasive wear, adhesive wear, delimitation wear, erosive wear, fatigue wear, fretting wear and corrosive and oxidation wear. The abrasive wear dominate under low load and sliding speed, while abrasive wear is converted to adhesive and further to delimitation with increase in normal load and sliding speed [64].

One of the phenomena used to reduce this conversion of abrasive wear to adhesive and further de-
Table 2. General properties of graphite, adapted from [34].

\begin{tabular}{ll}
\hline Bulk density & $1.3-1.95 \mathrm{~g} / \mathrm{cc}$ \\
Porosity & $0.7 \%-53 \%$ \\
Modulus of elasticity & $8-15 \mathrm{GPa}$ \\
Compressive strength & $20-200 \mathrm{MPa}$ \\
Thermal expansion coefficient & $1.2-8.2 \times 10^{-6} \mathrm{C}$ \\
Thermal conductivity & $25-470 \mathrm{~W} / \mathrm{mK}$ \\
Specific heat capacity & $710-8130 \mathrm{~J} / \mathrm{mK}$ \\
Electrical resistivity & $5 \times 10^{-6}-$ \\
& $30 \times 10^{-6} \Omega \mathrm{m}$ \\
\hline
\end{tabular}

limitation, is to reinforce solid lubricant into the sliding component of metal matrix composite as secondary reinforcement resulting in formation of new modifies generation of MMC's known as hybrid metal matrix composites. The self lubricating hybrid composites are the materials reinforced with solid lubricants such as graphite, $\mathrm{MoS}_{2}$, CNTs, and graphene that combines the strength, hardness and abrasion properties of MMC's with the self lubrication properties of solid lubricant. Graphite has been used as solid lubricant for fabrication of self lubricating hybrid composite of different alloys, metal and ceramics matrix composites of nickel [20,65-67], copper [17,21,22,68,69], Zinc [70-73], magnesium $[13,18,23,47,74,75]$, aluminum $[7,10-12,34-36,39$ $46,77]$, silver [78,79], silicon nitride [80,81], alumina [81] and bronze [82]. The solid lubricant graphite forms a thin transfer tribo-layer between the sliding interfaces that result in reduction of wear rate and friction coefficient. The formation of this transfer film between the sliding counterpart increases the seizure resistance and ensure that sliding interfaces are running under boundary lubrication without galling [28]. The major factors controlling the tribological behavior of graphite reinforced MMC's and HMMC's are composition, area fraction, thickness, formation and retention of transfer graphite tribo-layer; however graphite content in composite, sliding surfaces, surrounding environment and test condition can also be considered as important factors [28].

Aluminum and magnesium based MMC's and their HMMC's are widely used amongst the above stated alloys. For graphite reinforced $\mathrm{Al}$ and $\mathrm{Mg}$ based HMMC's, the main primary reinforcements used were $\mathrm{SiC}, \mathrm{B}_{4} \mathrm{C}, \mathrm{TiC}, \mathrm{Al}_{2} \mathrm{O}_{3}$, CNT, Tin, Zirconium and Flyash. The percentage contribution of these primary reinforcements in the conducted review is shown in Fig. 2. Among these materials, the combination of Silicon carbide and graphite (63\%) was the most reported hybrid reinforcement followed by 


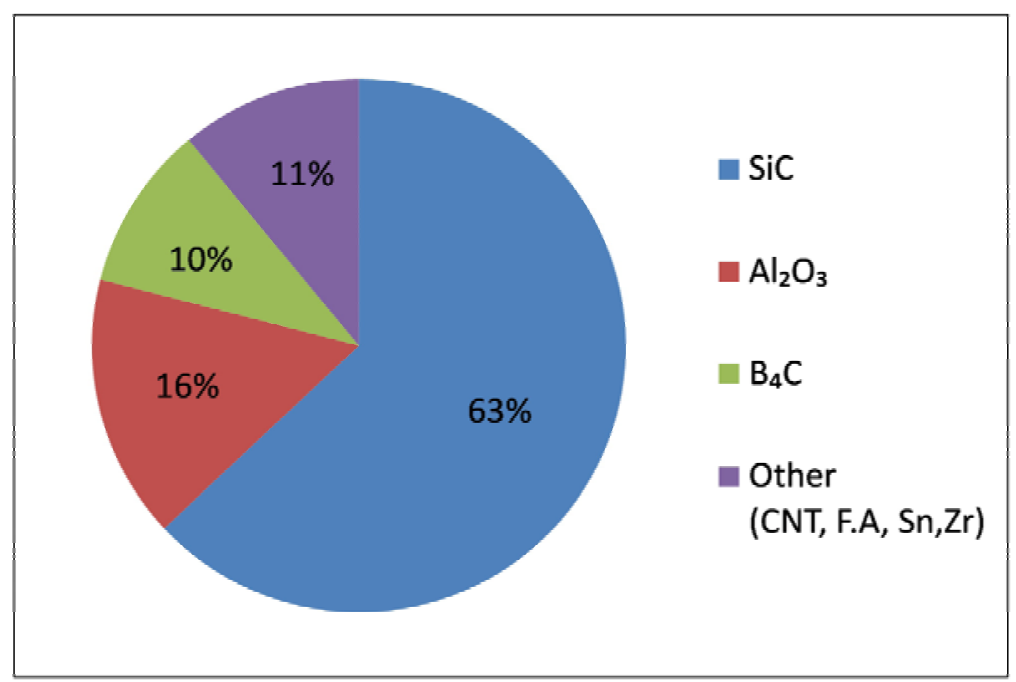

Fig. 2. Contribution (\%) of different primary reinforcements used for fabricating graphite reinforced Al and Mg based MMHCs reviewed in this report.

alumina and graphite (16\%) for fabrication of $\mathrm{Al}$ and Mg based self lubricating HMMC's.

The HMMC's reinforced with SiC and graphite (Gr) particles have attracted considerable attention due to their high strength combined with a low friction coefficient. The graphite content within specific limits increases the wear resistance of hybrid com- posites at the expense of material's overall strength, which is overcome by impregnating hard $\mathrm{SiC}$ in the hybrid Composites $[10,39]$. The mechanical and tribological properties of graphite reinforced aluminum and magnesium hybrid composites are further illustrated in below section and summarized in Table 3.

Table 3. The mechanical and tribological properties of aluminum and magnesium based self-lubricating hybrid composites reinforced with and without graphite.

\begin{tabular}{|c|c|c|c|c|c|c|c|}
\hline $\begin{array}{l}\text { Composi- } \\
\text { tion }\end{array}$ & $\begin{array}{l}\text { Fabrication } \\
\text { technique }\end{array}$ & $\begin{array}{l}\text { Tensile } \\
\text { strength } \\
\text { (MPa) }\end{array}$ & $\begin{array}{l}\text { Elongation } \\
(\%)\end{array}$ & Hardness & Wear rate & $\begin{array}{l}\text { Friction } \\
\text { coefficient } \\
(\mu)\end{array}$ & Ref \\
\hline Al 2024 & As-received alloy & - & - & $52 \mathrm{BHN}$ & $0.0111 \mathrm{~g}$ & 0.17 & [8] \\
\hline $\begin{array}{l}\text { Al 2024/5 } \\
\text { Gr }\end{array}$ & $\begin{array}{l}\text { Powder } \\
\text { metallurgy }\end{array}$ & - & - & $48 \mathrm{BHN}$ & $0.0119 \mathrm{~g}$ & 0.16 & \\
\hline $\begin{array}{l}\mathrm{Al} 2024 / 5 \\
\mathrm{Gr} / 5 \mathrm{SiC}\end{array}$ & $\begin{array}{l}\text { Powder } \\
\text { metallurgy }\end{array}$ & - & - & $53 \mathrm{BHN}$ & $0.0090 \mathrm{~g}$ & 0.18 & \\
\hline $\begin{array}{l}\text { Al 2024/5 } \\
\mathrm{Gr} / 10 \mathrm{SiC}\end{array}$ & $\begin{array}{l}\text { Powder } \\
\text { metallurgy }\end{array}$ & - & - & $56 \mathrm{BHN}$ & $0.0088 \mathrm{~g}$ & 0.24 & \\
\hline $\begin{array}{l}\mathrm{Al} 2024 / 5 \\
\mathrm{Gr} / 15 \mathrm{SiC}\end{array}$ & $\begin{array}{l}\text { Powder } \\
\text { metallurgy }\end{array}$ & - & - & $58 \mathrm{BHN}$ & $0.0079 \mathrm{~g}$ & 0.26 & \\
\hline $\begin{array}{l}\mathrm{Al} 2024 / 5 \\
\mathrm{Gr} / 20 \mathrm{SiC}\end{array}$ & $\begin{array}{l}\text { Powder } \\
\text { metallurgy }\end{array}$ & - & - & $63 \mathrm{BHN}$ & $0.0061 \mathrm{~g}$ & 0.32 & \\
\hline AISi10Mg & As-received alloy & 191.1 & - & 84.2 BHN & $\begin{array}{l}1.95 \mathrm{~mm}^{3} . \\
10^{-3} / \mathrm{m}^{2}\end{array}$ & 0.300 & [40] \\
\hline $\begin{array}{l}\text { AlSi10Mg } \\
/ 3 \mathrm{Gr}\end{array}$ & stir casting & 184.5 & - & 81.4 BHN & $\begin{array}{l}1.90 \mathrm{~mm}^{3} . \\
10^{-3} / \mathrm{m}\end{array}$ & 0.270 & \\
\hline $\begin{array}{l}\text { AlSi10Mg } \\
\text { /3FA/3Gr }\end{array}$ & stir casting & 204.6 & - & 84.4 BHN & $\begin{array}{l}1.80 \mathrm{~mm}^{3} . \\
10^{-3} / \mathrm{m}^{2}\end{array}$ & 0.250 & \\
\hline $\begin{array}{l}\text { AlSi10Mg } \\
\text { //6FA/3Gr }\end{array}$ & stir casting & 223.2 & - & $96.6 \mathrm{BHN}$ & $\begin{array}{l}1.50 \mathrm{~mm}^{3} . \\
10^{-3} / \mathrm{m}\end{array}$ & 0.242 & \\
\hline
\end{tabular}




\begin{tabular}{|c|c|c|c|c|c|c|}
\hline $\begin{array}{l}\text { AISi10Mg } \\
\text { /9FA/3Gr }\end{array}$ & stir casting & 238.7 & - & $101 \mathrm{BHN}$ & $\begin{array}{l}1.45 \mathrm{~mm}^{3} \text {. } \\
10^{-3} / \mathrm{m}\end{array}$ & 0.235 \\
\hline AZ91D & As-received alloy & 62.1 & - & $20.5 \mathrm{BHN}$ & $0.017 \mathrm{~g}$ & 0.36 \\
\hline $\begin{array}{l}\text { AZ91D/1.5 } \\
\mathrm{B}_{4} \mathrm{C}\end{array}$ & stir casting & 99.2 & - & $27.1 \mathrm{BHN}$ & $0.014 \mathrm{~g}$ & 0.37 \\
\hline $\begin{array}{l}\mathrm{AZ91D} / 1.5 \\
\mathrm{~B}_{4} \mathrm{C} / 1.5 \mathrm{Gr}\end{array}$ & stir casting & 86.3 & - & $22.5 \mathrm{BHN}$ & $0.013 \mathrm{~g}$ & 0.38 \\
\hline Al6061-T6 & As-received alloy & 271 & 12 & $105 \mathrm{HV}$ & $8.3 \mathrm{~m}^{3} / \mathrm{m}$ & - \\
\hline $\begin{array}{l}\text { Al 6061- } \\
\text { T6/8 }\end{array}$ & FSP & 185 & 9.1 & $132 \mathrm{HV}$ & $\begin{array}{l}2.2 \mathrm{~m}^{3} / \mathrm{m} \\
(900 \mathrm{rpm})\end{array}$ & - \\
\hline $\begin{array}{l}\mathrm{SiC} / 4 \mathrm{Al}_{2} \mathrm{O}_{3} \\
\mathrm{Al} 6061- \\
\mathrm{T} 6 / 8\end{array}$ & FSP & 137 & 6.4 & $124 \mathrm{HV}$ & $\begin{array}{l}3.2 \mathrm{~m}^{3} / \mathrm{m} \\
(1120 \mathrm{rpm})\end{array}$ & - \\
\hline $\begin{array}{l}\mathrm{SiC} / 4 \mathrm{Al}_{2} \mathrm{O}_{3} \\
\mathrm{Al} 6061- \\
\mathrm{T} 6 / 8\end{array}$ & FSP & 115 & 7.2 & $118 \mathrm{HV}$ & $\begin{array}{l}3.0 \mathrm{~m}^{3} / \mathrm{m} \\
(1400 \mathrm{rpm})\end{array}$ & - \\
\hline $\begin{array}{l}\mathrm{SiC} / 4 \mathrm{Al}_{2} \mathrm{O}_{3} \\
\mathrm{Al} 6061- \\
\mathrm{T} 6 / 8 \\
\mathrm{Si} / 1 \mathrm{Gr}\end{array}$ & FSP & 152 & 7.8 & $125 \mathrm{HV}$ & $\begin{array}{l}2.0 \mathrm{~m}^{3} / \mathrm{m} \\
(900 \mathrm{rpm})\end{array}$ & - \\
\hline $\begin{array}{l}\mathrm{SiC} / 4 \mathrm{Gr} \\
\mathrm{Al} 6061- \\
\mathrm{T} 6 / 8 \\
\mathrm{SiC} / 4 \mathrm{Gr}\end{array}$ & FSP & 112 & 5.9 & $120 \mathrm{HV}$ & $\begin{array}{l}2.4 \mathrm{~m}^{3} / \mathrm{m} \\
(1120 \mathrm{rpm})\end{array}$ & - \\
\hline $\begin{array}{l}\text { Al 6061- } \\
\text { T6/8 }\end{array}$ & FSP & 102 & 4.8 & $110 \mathrm{HV}$ & $\begin{array}{l}3.0 \mathrm{~m}^{3} / \mathrm{m} \\
(1400 \mathrm{rpm})\end{array}$ & - \\
\hline $\mathrm{SiC} / 4 \mathrm{Gr}$ & & & & & & \\
\hline Al 6101 & As-received alloy & 164 & 8.5 & $47.5 \mathrm{HV}$ & $\begin{array}{l}0.00081 \\
\mathrm{~mm}^{3} / \mathrm{Nm}\end{array}$ & 0.41 \\
\hline $\begin{array}{l}\text { Al 6101/ } \\
4 \mathrm{Gr}\end{array}$ & Liquid casting & 160 & 7.1 & $46.8 \mathrm{HV}$ & $\begin{array}{l}0.00021 \\
\mathrm{~mm}^{3} / \mathrm{Nm}\end{array}$ & 0.22 \\
\hline $\begin{array}{l}\text { Al 6101/ } \\
8 \mathrm{Gr}\end{array}$ & Liquid casting & 156 & 6.9 & $45.9 \mathrm{HV}$ & $\begin{array}{l}0.00029 \\
\mathrm{~mm}^{3} / \mathrm{Nm}\end{array}$ & 0.28 \\
\hline $\begin{array}{l}\text { Al 6101/ } \\
12 \mathrm{Gr}\end{array}$ & Liquid casting & 150 & 6.5 & $44.8 \mathrm{HV}$ & $\begin{array}{l}0.00034 \\
\mathrm{~mm}^{3} / \mathrm{Nm}\end{array}$ & 0.3 \\
\hline $\begin{array}{l}\text { Al 6101/ } \\
16 \mathrm{Gr}\end{array}$ & Liquid casting & 145 & 6 & $44.0 \mathrm{HV}$ & $\begin{array}{l}0.00039 \\
\mathrm{~mm}^{3} / \mathrm{Nm}\end{array}$ & 0.35 \\
\hline $\mathrm{Al} / 8 \mathrm{Gr}$ & Stir Casting & 120 & 12.8 & $30 \mathrm{HV}$ & $\begin{array}{l}15 \mathrm{Kg} / \mathrm{m} \\
10^{-9}\end{array}$ & 0.45 \\
\hline $\begin{array}{l}\mathrm{Al} / 8 \mathrm{Gr} / \\
0.2 \mathrm{Mg}\end{array}$ & Stir Casting & 145 & 11.6 & $37 \mathrm{HV}$ & $\begin{array}{l}13 \mathrm{Kg} / \mathrm{m} \\
10^{-9}\end{array}$ & 0.40 \\
\hline $\begin{array}{l}\mathrm{Al} / 8 \mathrm{Gr} / \\
0.4 \mathrm{Mg}\end{array}$ & Stir Casting & 168 & 9 & $56 \mathrm{HV}$ & $\begin{array}{l}6 \mathrm{Kg} / \mathrm{m} \\
10^{-9}\end{array}$ & 0.36 \\
\hline $\begin{array}{l}\mathrm{Al} / 8 \mathrm{Gr} / \\
0.6 \mathrm{Mg}\end{array}$ & Stir Casting & 184 & 7.8 & $67 \mathrm{HV}$ & $\begin{array}{l}4.2 \mathrm{Kg} / \mathrm{m} . \\
10^{-9}\end{array}$ & 0.33 \\
\hline $\begin{array}{l}\mathrm{Al} / 8 \mathrm{Gr} / \\
0.8 \mathrm{Mg}\end{array}$ & Stir Casting & 170 & 6.9 & $70 \mathrm{HV}$ & $\begin{array}{l}8 \mathrm{Kg} / \mathrm{m} \\
10^{-9}\end{array}$ & 0.31 \\
\hline $\begin{array}{l}\mathrm{Al} / 8 \mathrm{Gr} / \\
1 \mathrm{Mg}\end{array}$ & Stir Casting & 149 & 6.4 & $72 \mathrm{HV}$ & $\begin{array}{l}12.2 \mathrm{Kg} / \mathrm{m} . \\
10^{-9}\end{array}$ & 0.32 \\
\hline $\begin{array}{l}\mathrm{Al} / 6.3 \mathrm{Sn} / \\
1.6 \mathrm{Gr}\end{array}$ & Stir casting & 147.3 & 16.3 & $116 \mathrm{BHN}$ & - & 0.46 \\
\hline $\begin{array}{l}\mathrm{Al} / 6.3 \mathrm{Sn} / \\
2.4 \mathrm{Gr}\end{array}$ & Stir casting & 152.3 & 16.9 & $109 \mathrm{BHN}$ & - & 0.44 \\
\hline $\begin{array}{l}\mathrm{Al} / 6.3 \mathrm{Sn} / \\
3.4 \mathrm{Gr}\end{array}$ & Stir casting & 158.4 & 17.3 & $104 \mathrm{BHN}$ & - & 0.43 \\
\hline
\end{tabular}




\begin{tabular}{|c|c|c|c|c|c|c|}
\hline $\begin{array}{l}\mathrm{Al} / 6.3 \mathrm{Sn} / \\
5.6 \mathrm{Gr}\end{array}$ & Stir casting & 165.3 & 1.1 & $99.6 \mathrm{BHN}$ & - & 0.40 \\
\hline $\begin{array}{l}\mathrm{Al} / 6.3 \mathrm{Sn} / \\
8.4 \mathrm{Gr}\end{array}$ & Stir casting & 173.2 & 19.3 & $96.2 \mathrm{BHN}$ & - & 0.38 \\
\hline Al2024 & As-received alloy & $88^{*}$ & - & $73 \mathrm{HB}$ & $\begin{array}{l}0.0160 \\
\mathrm{~mm}^{3} / \mathrm{m}\end{array}$ & 0.35 \\
\hline $\begin{array}{l}\text { Al2024/ } \\
5 \mathrm{Gr}\end{array}$ & IPM & $50^{*}$ & - & $63 \mathrm{HB}$ & $\begin{array}{l}0.0015 \\
\mathrm{~mm}^{3} / \mathrm{m}\end{array}$ & 0.24 \\
\hline $\begin{array}{l}\text { Al2024/ } \\
10 \mathrm{Gr}\end{array}$ & IPM & $32.5^{\star}$ & - & $58 \mathrm{HB}$ & $\begin{array}{l}0.0035 \\
\mathrm{~mm}^{3} / \mathrm{m}\end{array}$ & 0.16 \\
\hline $\begin{array}{l}\text { Al2024/ } \\
15 \mathrm{Gr}\end{array}$ & IPM & $22.5^{\star}$ & - & $50 \mathrm{HB}$ & $\begin{array}{l}0.0042 \\
\mathrm{~mm} / \mathrm{m}\end{array}$ & 0.12 \\
\hline $\begin{array}{l}\text { Al2024/ } \\
20 \mathrm{Gr}\end{array}$ & IPM & $17.5^{\star}$ & - & $45 \mathrm{HB}$ & $\begin{array}{l}0.0058 \\
\mathrm{~mm}^{3} / \mathrm{m}\end{array}$ & 0.12 \\
\hline Al-Si10Mg & As-received alloy & 152.7 & - & $114 \mathrm{HV}$ & $\begin{array}{l}0.0049 \\
\mathrm{~mm}^{3} / \mathrm{m}\end{array}$ & 0.45 \\
\hline $\begin{array}{l}\mathrm{Al}-\mathrm{Si} 10 \mathrm{Mg} / \\
3 \mathrm{Al}_{2} \mathrm{O}_{3} / 3 \mathrm{Gr}\end{array}$ & Stir casting & 172.1 & - & $126 \mathrm{HV}$ & $\begin{array}{l}0.0030 \\
\mathrm{~mm}^{3} / \mathrm{m}\end{array}$ & 0.42 \\
\hline $\begin{array}{l}\mathrm{Al}-\mathrm{Si} 10 \mathrm{Mg} / \\
6 \mathrm{Al}_{2} \mathrm{O}_{3} / 3 \mathrm{Gr}\end{array}$ & Stir casting & 190 & - & $129 \mathrm{HV}$ & $\begin{array}{l}0.0029 \\
\mathrm{~mm}^{3} / \mathrm{m}\end{array}$ & 0.40 \\
\hline $\begin{array}{l}\mathrm{Al}-\mathrm{Si} 10 \mathrm{Mg} / \\
9 \mathrm{Al}_{2} \mathrm{O}_{3} / 3 \mathrm{Gr}\end{array}$ & Stir casting & 201 & - & $133 \mathrm{HV}$ & $\begin{array}{l}0.0027 \\
\mathrm{~mm}^{3} / \mathrm{m}\end{array}$ & 0.38 \\
\hline Al5083 & As-received alloy & 254 & - & 73.5 VHN & $43 \mu \mathrm{m}$ & - \\
\hline $\begin{array}{l}\text { Al 5083/ } \\
6 \mathrm{Gr}\end{array}$ & In-situ stir casting & 234 & - & 77.6 VHN & $38 \mu \mathrm{m}$ & - \\
\hline $\begin{array}{l}\text { Al 5083/ } \\
8 \mathrm{Gr}\end{array}$ & In-situ stir casting & 211 & - & 79.1 VHN & $32 \mu \mathrm{m}$ & - \\
\hline $\begin{array}{l}\text { Al 5083/ } \\
10 \mathrm{Gr}\end{array}$ & In-situ stir casting & 192 & - & $81.9 \mathrm{VHN}$ & $27 \mu \mathrm{m}$ & - \\
\hline $\begin{array}{l}\text { Al 5083/2 } \\
\text { MWCNT/ } \\
6 \mathrm{Gr}\end{array}$ & In-situ stir casting & 301 & - & 106.2 VHN & $37 \mu \mathrm{m}$ & - \\
\hline $\begin{array}{l}\text { Al } 5083 / 3 \\
\text { MWCNT/ } \\
6 \mathrm{Gr}\end{array}$ & In-situ stir casting & 319 & - & 118.0 VHN & $39 \mu \mathrm{m}$ & - \\
\hline $\begin{array}{l}\text { Al } 5083 / 4 \\
\text { MWCNT/ } \\
6 \mathrm{Gr}\end{array}$ & In-situ stir casting & 332 & - & 130.7 VHN & $40 \mu \mathrm{m}$ & - \\
\hline $\mathrm{Mg}$ & As-received Mg & - & - & $29 \mathrm{HV}$ & $0.0090 \mathrm{~g}$ & - \\
\hline $\mathrm{Mg} / 5 \mathrm{SiC}$ & $\begin{array}{l}\text { Powder } \\
\text { metallurgy }\end{array}$ & - & - & $50 \mathrm{HV}$ & $0.0077 \mathrm{~g}$ & - \\
\hline Mg/10SiC & $\begin{array}{l}\text { Powder } \\
\text { metallurgy }\end{array}$ & - & - & $80 \mathrm{HV}$ & $0.0062 \mathrm{~g}$ & - \\
\hline $\mathrm{Mg} / 5 \mathrm{Gr}$ & $\begin{array}{l}\text { Powder } \\
\text { metallurgy }\end{array}$ & - & - & $28 \mathrm{HV}$ & $0.0081 \mathrm{~g}$ & - \\
\hline $\mathrm{Mg} / 10 \mathrm{Gr}$ & $\begin{array}{l}\text { Powder } \\
\text { metallurgy }\end{array}$ & - & - & $22 \mathrm{HV}$ & $0.0084 \mathrm{~g}$ & - \\
\hline $\begin{array}{l}\mathrm{Mg} / 5 \mathrm{SiC} / \\
5 \mathrm{Gr}\end{array}$ & $\begin{array}{l}\text { Powder } \\
\text { metallurgy }\end{array}$ & - & - & $49 \mathrm{HV}$ & $0.0059 \mathrm{~g}$ & - \\
\hline $\begin{array}{l}\mathrm{Mg} / 5 \mathrm{SiC} / \\
10 \mathrm{Gr}\end{array}$ & $\begin{array}{l}\text { Powder } \\
\text { metallurgy }\end{array}$ & - & - & $47 \mathrm{HV}$ & $0.0081 \mathrm{~g}$ & - \\
\hline $\begin{array}{l}\mathrm{Mg} / 10 \mathrm{SiC} / \\
5 \mathrm{Gr}\end{array}$ & $\begin{array}{l}\text { Powder } \\
\text { metallurgy }\end{array}$ & - & - & $78 \mathrm{HV}$ & $0.0055 \mathrm{~g}$ & \\
\hline
\end{tabular}




\begin{tabular}{|c|c|c|c|c|c|c|}
\hline $\begin{array}{l}\mathrm{Mg} / 10 \mathrm{SiC} / \\
10 \mathrm{Gr}\end{array}$ & $\begin{array}{l}\text { Powder } \\
\text { metallurgy }\end{array}$ & - & - & $70 \mathrm{HV}$ & $0.0064 \mathrm{~g}$ & - \\
\hline $\mathrm{Al} / 5 \mathrm{SiC}$ & $\begin{array}{l}\text { Powder } \\
\text { metallurgy }\end{array}$ & - & - & $64.8 \mathrm{HB}$ & $314 \mathrm{mg}$ & \\
\hline $\begin{array}{l}\mathrm{Al} / 5 \mathrm{SiC} / \\
2 \mathrm{Gr}\end{array}$ & $\begin{array}{l}\text { Powder } \\
\text { metallurgy }\end{array}$ & - & - & $57.9 \mathrm{HB}$ & $265 \mathrm{mg}$ & \\
\hline $\begin{array}{l}\mathrm{Al} / 5 \mathrm{SiC} / \\
4 \mathrm{Gr}\end{array}$ & $\begin{array}{l}\text { Powder } \\
\text { metallurgy }\end{array}$ & - & - & $57.0 \mathrm{HB}$ & $165 \mathrm{mg}$ & \\
\hline $\begin{array}{l}\mathrm{Al} / 5 \mathrm{SiC} / \\
6 \mathrm{Gr}\end{array}$ & $\begin{array}{l}\text { Powder } \\
\text { metallurgy }\end{array}$ & - & - & $55.5 \mathrm{HB}$ & $60 \mathrm{mg}$ & \\
\hline $\begin{array}{l}\mathrm{Al} / 5 \mathrm{SiC} / \\
8 \mathrm{Gr}\end{array}$ & $\begin{array}{l}\text { Powder } \\
\text { metallurgy }\end{array}$ & - & - & $54.9 \mathrm{HB}$ & $80 \mathrm{mg}$ & \\
\hline $\begin{array}{l}\text { As extru- } \\
\operatorname{ded} \text { AZ91 }\end{array}$ & As-received alloy & 200 & 17.5 & - & - & - \\
\hline $\begin{array}{l}\text { As extru- } \\
\text { ded AZ91/ } \\
5 \mathrm{Gr}\end{array}$ & Stir casting & 250 & 6.1 & - & - & - \\
\hline $\begin{array}{l}\text { As extru- } \\
\text { ded AZ91/ } \\
10 \mathrm{Gr}\end{array}$ & Stir casting & 240 & 2.6 & - & - & - \\
\hline $\begin{array}{l}\text { As extru- } \\
\text { ded AZ91/ } \\
15 \mathrm{Gr}\end{array}$ & Stir casting & 200 & 1.25 & - & - & - \\
\hline $\begin{array}{l}\text { As extru- } \\
\text { ded AZ91/ } \\
20 \mathrm{Gr}\end{array}$ & Stir casting & 175 & 1 & - & - & - \\
\hline Al-Si alloy & As-received alloy & - & - & $53.7 \mathrm{BHN}$ & $230 \mu \mathrm{m}^{\#}$ & 0.83 \\
\hline $\begin{array}{l}\text { Al-Si alloy/ } \\
2.5 \mathrm{Gr}\end{array}$ & SASC & - & - & 47.5BHN & $196 \mu \mathrm{m}$ & 0.81 \\
\hline $\begin{array}{l}\text { Al-Si alloy/ } \\
5 \mathrm{Gr}\end{array}$ & SASC & - & - & 41.2BHN & $154 \mu \mathrm{m}$ & 0.80 \\
\hline $\begin{array}{l}\text { Al-Si alloy/ } \\
7.5 \mathrm{Gr}\end{array}$ & SASC & - & - & $36 \mathrm{BHN}$ & $104 \mu \mathrm{m}$ & 0.79 \\
\hline $\mathrm{Al} / 5 \mathrm{SiC}$ & $\begin{array}{l}\text { Powder } \\
\text { metallurgy }\end{array}$ & - & - & $55 \mathrm{BHN}$ & $0.0078 \mathrm{~g}$ & 0.148 \\
\hline $\begin{array}{l}\mathrm{Al} / 5 \mathrm{SiC} / \\
5 \mathrm{Gr}\end{array}$ & $\begin{array}{l}\text { Powder } \\
\text { metallurgy }\end{array}$ & - & - & $53 \mathrm{BHN}$ & $0.0069 \mathrm{~g}$ & 0.144 \\
\hline $\begin{array}{l}\mathrm{Al} / 5 \mathrm{SiC} / \\
10 \mathrm{Gr}\end{array}$ & $\begin{array}{l}\text { Powder } \\
\text { metallurgy }\end{array}$ & - & - & $51 \mathrm{BHN}$ & $0.0061 \mathrm{~g}$ & 0.141 \\
\hline Al 6082 & As-received alloy & - & - & $43 \mathrm{HRW}$ & $\begin{array}{l}0.7370 \\
\mathrm{~mm}^{3} / \mathrm{m}\end{array}$ & - \\
\hline $\begin{array}{l}\text { Al 6082/ } \\
10 \mathrm{SiC}\end{array}$ & stir casting & - & - & $54 \mathrm{HRW}$ & $\begin{array}{l}0.5262 \\
\mathrm{~mm} / \mathrm{m}\end{array}$ & - \\
\hline $\begin{array}{l}\text { Al } 6082 / \\
5 \mathrm{SiC} / 5 \mathrm{Gr}\end{array}$ & stir casting & - & - & $49 \mathrm{HRW}$ & $\begin{array}{l}0.3964 \\
\mathrm{~mm}^{3} / \mathrm{m}\end{array}$ & - \\
\hline $\begin{array}{l}\text { Al 6061/ } \\
2 \mathrm{SiC}\end{array}$ & $\begin{array}{l}\text { Powder } \\
\text { metallurgy }\end{array}$ & - & - & $68 \mathrm{HRB}$ & $40000 \mu \mathrm{m}^{3}$ & 0.371 \\
\hline $\begin{array}{l}\text { Al 6061/ } \\
2 \mathrm{SiC} / 2 \mathrm{Gr}\end{array}$ & $\begin{array}{l}\text { Powder } \\
\text { metallurgy }\end{array}$ & - & - & $58 \mathrm{HRB}$ & $23000 \mu \mathrm{m}^{3}$ & 0.340 \\
\hline $\begin{array}{l}\text { Al } 6061 / \\
2 \mathrm{SiC} / 5 \mathrm{Gr}\end{array}$ & $\begin{array}{l}\text { Powder } \\
\text { metallurgy }\end{array}$ & - & - & $45 \mathrm{HRB}$ & $50000 \mu \mathrm{m}^{3}$ & 0.358 \\
\hline $\begin{array}{l}\text { Al } 6061 / \\
2 \mathrm{SiC} / 7 \mathrm{Gr}\end{array}$ & $\begin{array}{l}\text { Powder } \\
\text { metallurgy }\end{array}$ & - & - & $40 \mathrm{HRB}$ & $63000 \mu \mathrm{m}^{3}$ & 0.410 \\
\hline
\end{tabular}




\begin{tabular}{|c|c|c|c|c|c|c|}
\hline Al6061 & As-received alloy & - & - & - & $\begin{array}{l}300 \\
\mathrm{~mm}^{3} .10^{3} / \mathrm{m}\end{array}$ & \\
\hline $\begin{array}{l}\text { Al 6061/ } \\
9 \mathrm{Gr}\end{array}$ & $\begin{array}{l}\text { Powder } \\
\text { metallurgy }\end{array}$ & - & - & $50 \mathrm{HBN}$ & $\begin{array}{l}3000 \\
\mathrm{~mm}^{3} .10^{3} / \mathrm{m}\end{array}$ & - \\
\hline $\begin{array}{l}\text { Al } 6061 / 9 \\
\mathrm{Gr} / 20 \mathrm{SiC}\end{array}$ & $\begin{array}{l}\text { Powder } \\
\text { metallurgy }\end{array}$ & - & - & $65 \mathrm{HBN}$ & $\begin{array}{l}10 \\
\mathrm{~mm}^{3} .10^{3} / \mathrm{m}\end{array}$ & - \\
\hline $\begin{array}{l}\text { Al } 6061 / 9 \\
\mathrm{Gr} / 20 \mathrm{SiC}\end{array}$ & $\begin{array}{l}\text { Powder } \\
\text { metallurgy }\end{array}$ & - & - & $75 \mathrm{HBN}$ & $\begin{array}{l}45 \\
\mathrm{~mm}^{3} .10^{3} / \mathrm{m}\end{array}$ & - \\
\hline Al 2024 & As-received alloy & - & - & $84 \mathrm{BHN}$ & - & - \\
\hline $\begin{array}{l}\text { Al 2024/ } \\
5 \text { SiC }\end{array}$ & $\begin{array}{l}\text { Powder } \\
\text { metallurgy }\end{array}$ & - & - & $87 \mathrm{BHN}$ & $0.01625 \mathrm{~g}$ & - \\
\hline $\begin{array}{l}\mathrm{Al} 2024 / \\
5 \mathrm{SiC} / 5 \mathrm{Gr}\end{array}$ & $\begin{array}{l}\text { Powder } \\
\text { metallurgy }\end{array}$ & - & - & $83 \mathrm{BHN}$ & $0.01440 \mathrm{~g}$ & - \\
\hline $\begin{array}{l}\text { Al 2024/ } \\
5 \mathrm{SiC} / 10 \mathrm{Gr}\end{array}$ & $\begin{array}{l}\text { Powder } \\
\text { metallurgy }\end{array}$ & - & - & $81 \mathrm{BHN}$ & $0.01170 \mathrm{~g}$ & \\
\hline Al 6061 & As-received alloy & - & - & - & - & - \\
\hline $\begin{array}{l}\text { Al } 6061 / \\
10 \mathrm{SiC}\end{array}$ & SSPM & - & - & $62 \mathrm{HV}$ & $0.09 \mathrm{~g}$ & 1.10 \\
\hline $\begin{array}{l}\mathrm{Al} 6061 / 10 \\
\mathrm{SiC} / 2 \mathrm{Gr}\end{array}$ & SSPM & - & - & $59 \mathrm{HV}$ & $0.38 \mathrm{~g}$ & 1.05 \\
\hline $\begin{array}{l}\mathrm{Al} 6061 / 10 \\
\mathrm{SiC} / 5 \mathrm{Gr}\end{array}$ & SSPM & - & - & $55 \mathrm{HV}$ & $1.09 \mathrm{~g}$ & 0.88 \\
\hline $\begin{array}{l}\text { Al } 6061 / 10 \\
\mathrm{SiC} / 8 \mathrm{Gr}\end{array}$ & SSPM & - & - & $49 \mathrm{HV}$ & $0.71 \mathrm{~g}$ & 0.89 \\
\hline Al6106 & As-received alloy & - & - & $55 \mathrm{BHN}$ & $\begin{array}{l}0.040 \\
\mathrm{~mm}^{3} / \mathrm{m}\end{array}$ & 0.92 \\
\hline $\begin{array}{l}\text { Al6106/ } \\
9 \mathrm{Gr}\end{array}$ & IPM & - & - & $49 \mathrm{BHN}$ & $\begin{array}{l}0.085 \\
\mathrm{~mm}^{3} / \mathrm{m}\end{array}$ & 0.60 \\
\hline $\begin{array}{l}\text { Al6106/ } \\
9 \mathrm{Gr} / 10 \mathrm{SiC}\end{array}$ & IPM & - & - & $63 \mathrm{BHN}$ & $\begin{array}{l}0.019 \\
\mathrm{~mm}^{3} / \mathrm{m}\end{array}$ & 0.58 \\
\hline $\begin{array}{l}\text { Al6106/ } \\
9 \mathrm{Gr} / 20 \mathrm{SiC}\end{array}$ & IPM & - & - & $69 \mathrm{BHN}$ & $\begin{array}{l}0.010 \\
\mathrm{~mm}^{3} / \mathrm{m}\end{array}$ & 0.55 \\
\hline $\begin{array}{l}\text { Al6106/ } \\
9 \mathrm{Gr} / 30 \mathrm{SiC}\end{array}$ & IPM & - & - & $73 \mathrm{BHN}$ & $\begin{array}{l}0.018 \\
\mathrm{~mm} / \mathrm{m}\end{array}$ & 0.54 \\
\hline $\begin{array}{l}\text { Al6106/ } \\
9 \mathrm{Gr} / 40 \mathrm{SiC}\end{array}$ & IPM & - & - & $75 \mathrm{BHN}$ & $\begin{array}{l}0.023 \\
\mathrm{~mm}^{3} / \mathrm{m}\end{array}$ & 0.56 \\
\hline Al 6061 & As-received alloy & - & - & - & - & - \\
\hline Al 6061/ & IPM & - & - & 91 & $\begin{array}{l}10 \mathrm{~mm}^{3 /} \\
\mathrm{m} .10^{-3}\end{array}$ & 0.63 \\
\hline $\begin{array}{l}\mathrm{Al} 6061 / 30 \\
\mathrm{SiC} / 3 \mathrm{Gr}\end{array}$ & IPM & - & - & 89 & $\begin{array}{l}8.5 \mathrm{~mm}^{3} / \\
\mathrm{m} .10^{-3}\end{array}$ & 0.60 \\
\hline $\begin{array}{l}\mathrm{Al} 6061 / 30 \\
\mathrm{SiC} / 5 \mathrm{Gr}\end{array}$ & IPM & - & - & 87.5 & $\begin{array}{l}7.5 \mathrm{~mm}^{3} / \\
\mathrm{m} .10^{-3}\end{array}$ & 0.58 \\
\hline $\begin{array}{l}\mathrm{Al} 6061 / 30 \\
\mathrm{SiC} / 9 \mathrm{Gr}\end{array}$ & IPM & - & - & 85 & $\begin{array}{l}2 \mathrm{~mm}^{3 /} \\
\mathrm{m} \cdot 10^{-3}\end{array}$ & 0.52 \\
\hline $\begin{array}{l}\text { Al } 6061 / 30 \\
\mathrm{SiC} / 13 \mathrm{Gr}\end{array}$ & IPM & - & - & 82.5 & $\begin{array}{l}6 \mathrm{~mm}^{3} / \\
\mathrm{m} \cdot 10^{-3}\end{array}$ & 0.50 \\
\hline $\begin{array}{l}\mathrm{Mg} / 10 \\
\mathrm{GNF} / \\
\text { Alumina }\end{array}$ & infiltration method & 240 & - & $142 \mathrm{HV}$ & - & - \\
\hline $\begin{array}{l}\text { Mg/ } 15 \\
\text { GNF/ }\end{array}$ & infiltration method & 238 & - & $136 \mathrm{HV}$ & - & - \\
\hline
\end{tabular}

Alumina 


\begin{tabular}{|c|c|c|c|c|c|c|}
\hline $\begin{array}{l}\mathrm{Mg} / 20 \\
\text { GNF/ } \\
\text { Alumina }\end{array}$ & infiltration method & 233 & - & $120 \mathrm{HV}$ & - & - \\
\hline $\begin{array}{l}\mathrm{Mg} / 10 \\
\text { Alumina }\end{array}$ & infiltration method & 236 & - & $130 \mathrm{HV}$ & - & - \\
\hline $\begin{array}{l}\mathrm{Mg} / 15 \\
\text { Alumina }\end{array}$ & infiltration method & 237 & - & $135 \mathrm{HV}$ & - & - \\
\hline $\begin{array}{l}\mathrm{Mg} / 20 \\
\text { Alumina }\end{array}$ & infiltration method & 239 & - & $140 \mathrm{HV}$ & - & - \\
\hline AA7075 & As-received alloy & - & - & $116 \mathrm{BHN}$ & $\begin{array}{l}0.0175 \\
\mathrm{mg} / \mathrm{Nm}\end{array}$ & 0.61 \\
\hline $\begin{array}{l}\text { AA7075/ } \\
5 \mathrm{Gr}\end{array}$ & Liquid casting & - & - & $112 \mathrm{BHN}$ & $\begin{array}{l}0.0051 \\
\mathrm{mg} / \mathrm{Nm}\end{array}$ & 0.48 \\
\hline $\begin{array}{l}\text { AA7075/ } \\
10 \mathrm{Gr}\end{array}$ & Liquid casting & - & - & $100 \mathrm{BHN}$ & $\begin{array}{l}0.0065 \\
\mathrm{mg} / \mathrm{Nm}\end{array}$ & 0.49 \\
\hline $\begin{array}{l}\text { AA7075/ } \\
15 \mathrm{Gr}\end{array}$ & Liquid casting & - & - & $95 \mathrm{BHN}$ & $\begin{array}{l}0.0090 \\
\mathrm{mg} / \mathrm{Nm}\end{array}$ & 0.54 \\
\hline $\begin{array}{l}\text { AA7075/ } \\
20 \mathrm{Gr}\end{array}$ & Liquid casting & - & - & $90 \mathrm{BHN}$ & $\begin{array}{l}0.0090 \\
\mathrm{mg} / \mathrm{Nm}\end{array}$ & 0.58 \\
\hline AZ91 & As-received alloy & - & - & - & - & - \\
\hline $\begin{array}{l}\mathrm{AZ91/1} \\
\mathrm{SiC} / 1 \mathrm{Gr}\end{array}$ & Vortex method & - & - & $62.8 \mathrm{HV}$ & $\begin{array}{l}0.0045 \\
\mathrm{~mm}^{3} / \mathrm{Km}\end{array}$ & - \\
\hline $\begin{array}{l}\mathrm{AZ91/2} \\
\mathrm{SiC} / 2 \mathrm{Gr}\end{array}$ & Vortex method & - & - & $64.3 \mathrm{HV}$ & $\begin{array}{l}0.0041 \\
\mathrm{~mm}^{3} / \mathrm{Km}\end{array}$ & - \\
\hline $\begin{array}{l}\mathrm{AZ91/3} \\
\mathrm{SiC} / 3 \mathrm{Gr}\end{array}$ & Vortex method & - & - & $66.2 \mathrm{HV}$ & $\begin{array}{l}0.0037 \\
\mathrm{~mm}^{3} / \mathrm{Km}\end{array}$ & - \\
\hline $\begin{array}{l}\text { Al6061/ } \\
2 \mathrm{Gr}\end{array}$ & Stir casting & - & - & $51 \mathrm{BHN}$ & $0.0040 \mathrm{~g}$ & - \\
\hline $\begin{array}{l}\text { Al6061/ } \\
2 \mathrm{Gr} / 2 \mathrm{Zr}\end{array}$ & Stir casting & - & - & $60 \mathrm{BHN}$ & $0.0035 \mathrm{~g}$ & - \\
\hline $\begin{array}{l}\text { Al6061/ } \\
2 \mathrm{Gr} / 4 \mathrm{Zr}\end{array}$ & Stir casting & - & - & $65 \mathrm{BHN}$ & $0.0030 \mathrm{~g}$ & - \\
\hline $\begin{array}{l}\text { Al6061/ } \\
2 \mathrm{Gr} / 6 \mathrm{Zr}\end{array}$ & Stir casting & - & - & $65 \mathrm{BHN}$ & $0.0029 \mathrm{~g}$ & - \\
\hline $\begin{array}{l}\text { Al6061/ } \\
2 \mathrm{Gr} / 8 \mathrm{Zr}\end{array}$ & Stir casting & - & - & $59 \mathrm{BHN}$ & $0.0027 \mathrm{~g}$ & - \\
\hline $\begin{array}{l}\text { Al6061/ } \\
2 \mathrm{Gr} / 10 \mathrm{Zr}\end{array}$ & Stir casting & - & - & $60 \mathrm{BHN}$ & $0.0029 \mathrm{~g}$ & - \\
\hline Al(LM25) & As-received alloy & - & - & 87 VHN & $0.0030 \mathrm{~g}$ & - \\
\hline $\begin{array}{l}\mathrm{Al}(\mathrm{LM} 25) / \\
3 \mathrm{~B}_{4} \mathrm{C} / 3 \mathrm{Gr}\end{array}$ & Stir Casting & - & - & $110 \mathrm{VHN}$ & $0.0028 \mathrm{~g}$ & - \\
\hline $\begin{array}{l}\mathrm{Al}(\mathrm{LM} 25) / \\
3 \mathrm{~B}_{4} \mathrm{C} / 3 \mathrm{Gr}\end{array}$ & Stir Casting & - & - & 119 VHN & $0.0023 \mathrm{~g}$ & - \\
\hline $\begin{array}{l}\mathrm{Al}(\mathrm{LM} 25) / \\
3 \mathrm{~B}_{4} \mathrm{C} / 3 \mathrm{Gr}\end{array}$ & Stir Casting & - & - & $128 \mathrm{VHN}$ & $0.0018 \mathrm{~g}$ & - \\
\hline $\begin{array}{l}\mathrm{Al}(\mathrm{LM} 25) / \\
3 \mathrm{~B}_{4} \mathrm{C} / 3 \mathrm{Gr}\end{array}$ & Stir Casting & - & - & $140 \mathrm{VHN}$ & $0.0016 \mathrm{~g}$ & - \\
\hline $\mathrm{Al}$ & As-received Al & - & - & $33 \mathrm{HV}$ & $3 \mathrm{~g} \cdot 10^{-6} / \mathrm{m}$ & - \\
\hline $\mathrm{Al} / 1 \mathrm{Gr}$ & $\begin{array}{l}\text { Powder } \\
\text { metallurgy }\end{array}$ & - & - & $39 \mathrm{HV}$ & $1 \mathrm{~g} .10^{-6} / \mathrm{m}$ & - \\
\hline $\mathrm{Al} / 2 \mathrm{Gr}$ & $\begin{array}{l}\text { Powder } \\
\text { metallurgy }\end{array}$ & - & - & $45 \mathrm{HV}$ & $\begin{array}{l}0.7 \mathrm{~g} . \\
10^{-6} / \mathrm{m}\end{array}$ & - \\
\hline $\mathrm{Al} / 3 \mathrm{Gr}$ & $\begin{array}{l}\text { Powder } \\
\text { metallurgy }\end{array}$ & - & - & $55 \mathrm{HV}$ & $\begin{array}{l}0.5 \mathrm{~g} . \\
10^{-6} / \mathrm{m}\end{array}$ & \\
\hline
\end{tabular}




$\begin{array}{llllll}\mathrm{Al} / 4 \mathrm{Gr} & \text { Powder } & - & - & 40 \mathrm{HV} & 0.4 \mathrm{~g} . \\ & \text { metallurgy } & & & & 10^{-6 / \mathrm{m}} \\ \mathrm{Al} / 5 \mathrm{Gr} & \text { Powder } & - & - & 29 \mathrm{HV} & 0.3 \mathrm{~g} . \\ & \text { metallurgy } & & & & 10^{-6 / \mathrm{m}} \\ \mathrm{A} 356 / & \mathrm{MCP} & - & - & - & 1.199 \mathrm{~mm}^{3} .- \\ 10 \mathrm{SiC} & & & & 10^{-5} / \mathrm{Nm}^{3} \\ \mathrm{~A} 356 / 10 & \text { MCP } & - & - & - & 0.822 \mathrm{~mm}^{3} .- \\ \mathrm{SiC} / 1 \mathrm{Gr} & & & & 10^{-5} / \mathrm{Nm}^{3} \\ \mathrm{~A} 356 / 10 & \mathrm{MCP} & - & - & - & 1.263 \mathrm{~mm}^{3} . \\ \mathrm{SiC} / 3 \mathrm{Gr} & & & & & 10^{-5} / \mathrm{Nm}^{-}\end{array}$

(\#) represents wear loss of sample pins was measured in terms of height loss in microns with accuracy of $1 \mu \mathrm{m}$. $\left(^{*}\right)$ mark values show bending strength in MPa.(á) marked values are representing specific density. (-) mark represent property not calculated/reported in particular article. MCP denotes modified compo casting procedure; IPM denotes In -situ powder metallurgy; SSPM denotes semi solid powder densification; SASC denotes stir and squeeze casting.

\section{MECHANICAL AND MICRO STRUCTURAL PROPERTIES OF HYBRID COMPOSITES}

I.Aatthisugan et al. [18] experimentally studied the micro structural and mechanical behavior of unreinforced AZ91D magnesium alloy, AZ91D-B $\mathrm{B}_{4}$ composites andAZ91D-B $\mathrm{B}_{4}$-Gr hybrid composites fabricated by stir casting. They have reported an increase in density with addition of $B_{4} C$ in AZ91D and further it slightly increased with embedment of graphite as AZ91D-B $\mathrm{C}-\mathrm{Gr}$ hybrid composite. The porosity showed lower value for $A Z 91 D-B_{4} C$ composite and further lowest value for AZ91D-B $\mathrm{C}-\mathrm{Gr}$ hybrid composite. This decrease in porosity was due to uniform stirring speed and also size of reinforcements. The results also revealed that AZ91D$\mathrm{B}_{4} \mathrm{C}$ composite and AZ91D- $\mathrm{B}_{4} \mathrm{C}-\mathrm{Gr}$ hybrid composite had superior hardness and ultimate tensile strength (UTS) than base AZ91D magnesium alloy. The decrease in hardness and UTS values was reported for hybrid composite with respect to AZ91D$\mathrm{B}_{4} \mathrm{C}$ composite as shown in Fig. 3. Metallographic analysis shows uniformly distribution of $\mathrm{B}_{4} \mathrm{C}$ and graphite particles throughout the AZ91D matrix phase with lack of cracks as shown in Fig. 4. S.Srivastava et al. [83] developed AM50/Graphite nano fiber (GNF)/Al $\mathrm{O}_{3}$ hybrid composites by infiltration method and experimentally assessed the influence of GNF on the mechanical behavior of $\mathrm{AM} 50 / \mathrm{Al}_{2} \mathrm{O}_{3}$ composite system. The AM50/GNF/Al $\mathrm{O}_{3}$ hybrid composite exhibited the superior mechanical properties than base alloy; however the mechanical properties such as hardness, tensile strength and compressive strength of hybrid composite were inferior than AM50/ $\mathrm{Al}_{2} \mathrm{O}_{3}$ composite at same reinforcement composite $(10,15,20 \mathrm{wt} . \%)$. The reason for this decrease in properties was agglomeration tendency of GNF which weaken the bonding between magnesium matrix and alumina.

D.S.R Smart et al. [34] characterized the mechanical behavior of graphite reinforced and graphite/MWCNT (multi wall carbon nanotubes) reinforced Al5083 hybrid composites. It has been revealed that micro hardness of Al5083 alloy was increased in small value with addition of graphite and increased in large extent with further addition of MWCNT. This increase in hardness was observed due to excellent strength to weight ratio of MWCNT and thermal mismatch caused by difference in co-efficient of thermal expansion of Al5083 and MWCNT. Tensile strength was decreased with an addition of graphite; however it was increased with further addition of CNT in Al5083-Gr composite. The percentage elongation was also less for Al5083 composite with CNT and graphite reinforcement. It was concluded that graphite reinforcement increased the wear resistance of hybrid composite while decreasing the over-

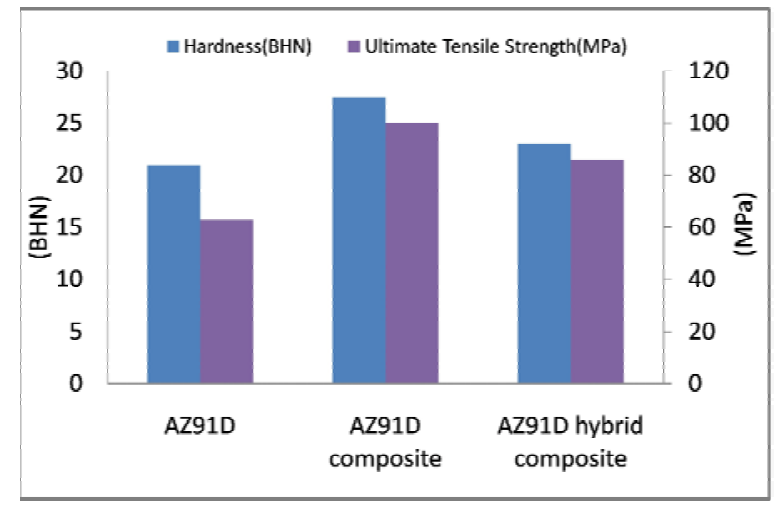

Fig. 3. Variation of hardness and ultimate tensile strength of AZ91D, AZ91D/B 4 composite and $\mathrm{AZ91D/B}{ }_{4} \mathrm{C} / \mathrm{Gr}$ hybrid composite, adapted from [18]. 


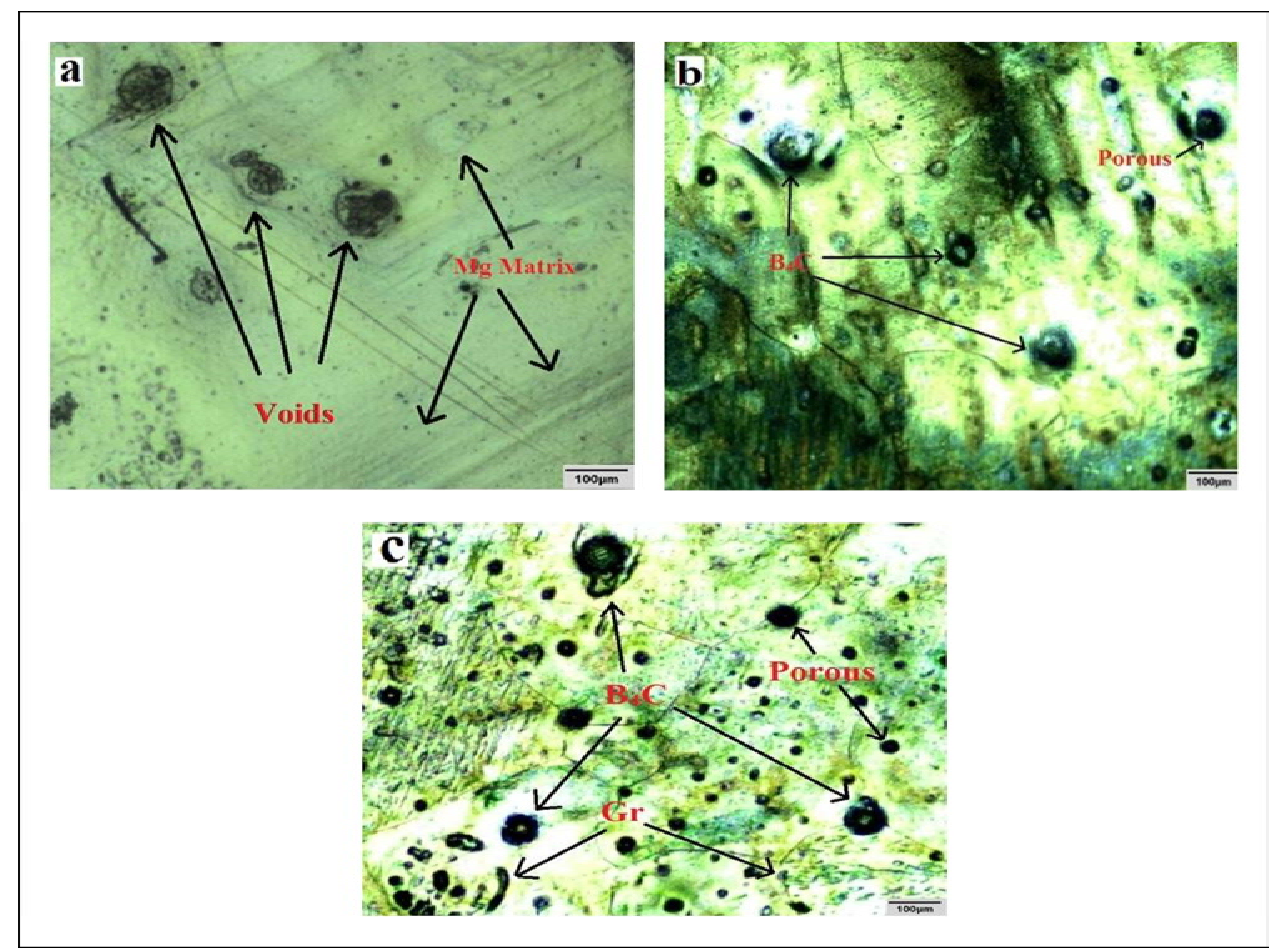

Fig. 4. Optical micrographs of the produced composites: (a) AZ91D, (b) AZ91D/1.5\% $B_{4} C$, (c) AZ91D/1.5\% $\mathrm{B}_{4} \mathrm{C} / 1.5 \% \mathrm{Gr}$, reprinted with permission from I. Aatthisugan, A.R. Rose and D.S. Jebadurai // J. Magnes. Alloys 5(2017)20, under the CC BY-NC-ND 4.0 license (http://creativecommons.org/licenses/by-nc-nd/ 4.0/).

all strength and to counter this decline in strength MWCNT particles were added. P. Ravinder et al. [35] fabricated hybrid Al2024/SiC/Gr composites and examined the mechanical behavior of fabricated hybrid composite where graphite was used as a solid lubricant. It has been revealed that hardness and density were increased with an addition of $100 \mathrm{~nm}$ size SiC in Al2024 alloy while hardness and density decrease with further addition of $40-50 \mathrm{~nm}$ graphite in Al2024-SiC composite. This decrease in mechanical properties was due to low hardness of graphite particles and uniform distribution of graphite in hybrid nano composite. The microstructural investigation shown in Fig. 5 illustrated deep permanent grooves, micropits and fracture of the oxide layer for Al2024 matrix; however smooth grooves at surface of $\mathrm{Al} / 5 \% \mathrm{SiC} / 10 \% \mathrm{Gr}$ hybrid composite represent high wear resistance.

L. Jinfeny et al. [36] examined the effect of graphite particle reinforcement on the mechanical behavior of hybrid $\mathrm{Al} / \mathrm{SiC} / \mathrm{Gr}$ composite. The results showed that tensile strength was reduced from 510 to 420MPa and elastic modulus was reduced from 172 to $166 \mathrm{GPa}$ with addition of $5 \mathrm{wt} . \%$ of graphite of 1 microns. The above trend of reduction in mechanical properties was also followed with an increase in size of graphite; However, due to presence of graph- ite in hybrid $\mathrm{Al} / \mathrm{SiC} / \mathrm{Gr}$ composite, wear rate and coefficient of friction of hybrid composite was decreased. Moreover, coarse graphite enhanced the integrity of tribo layer as shown in Fig. 6a resulted in higher wear resistance as compared to fine graphite particles. EDS analysis of worn surface in Fig. $6 \mathrm{~b}$ illustrated the presence of iron and oxygen, which further inferred that tribo lubrication layer was composed of iron oxides, $\mathrm{SiC}$ and fine aluminum particles.

A. Baradeswaran et al. [56] experimentally investigated the mechanical and microstructural properties of $\mathrm{Al} 7075 / \mathrm{Al}_{2} \mathrm{O}_{3} / 5 \mathrm{wt} . \% \mathrm{Gr}$ hybrid composites were improved with an increase in content of $\mathrm{Al}_{2} \mathrm{O}_{3}$ $(2,4,6,8 \mathrm{wt} . \%)$. The hardness was decreased with graphite reinforcement and to counter this decrease in hardness $\mathrm{Al}_{2} \mathrm{O}_{3}$ hard ceramic particles were added and these particles acted as obstacle to the movement of dislocations. D. Aruri et al. [38] investigated that mechanical properties of Al6061-T6/SiC/Gr \& Al6061- $\mathrm{T} 6 / \mathrm{SiC} / \mathrm{Al}_{2} \mathrm{O}_{3}$ hybrid composite were decreased with increase in tool rotational speed fabricated via friction stir processing (FSP). The results illustrated that $\mathrm{Al} / \mathrm{SiC}$ composite reinforced with 4 wt.\% Gr had high mechanical properties than Al/ $\mathrm{SiC}$ composite reinforced with 4 wt. $\% \mathrm{Al}_{2} \mathrm{O}_{3}$. The Al6061-T6/SiC/Gr and Al6061-T6/SiC/ $/ \mathrm{Al}_{2} \mathrm{O}_{3}$ hybrid 

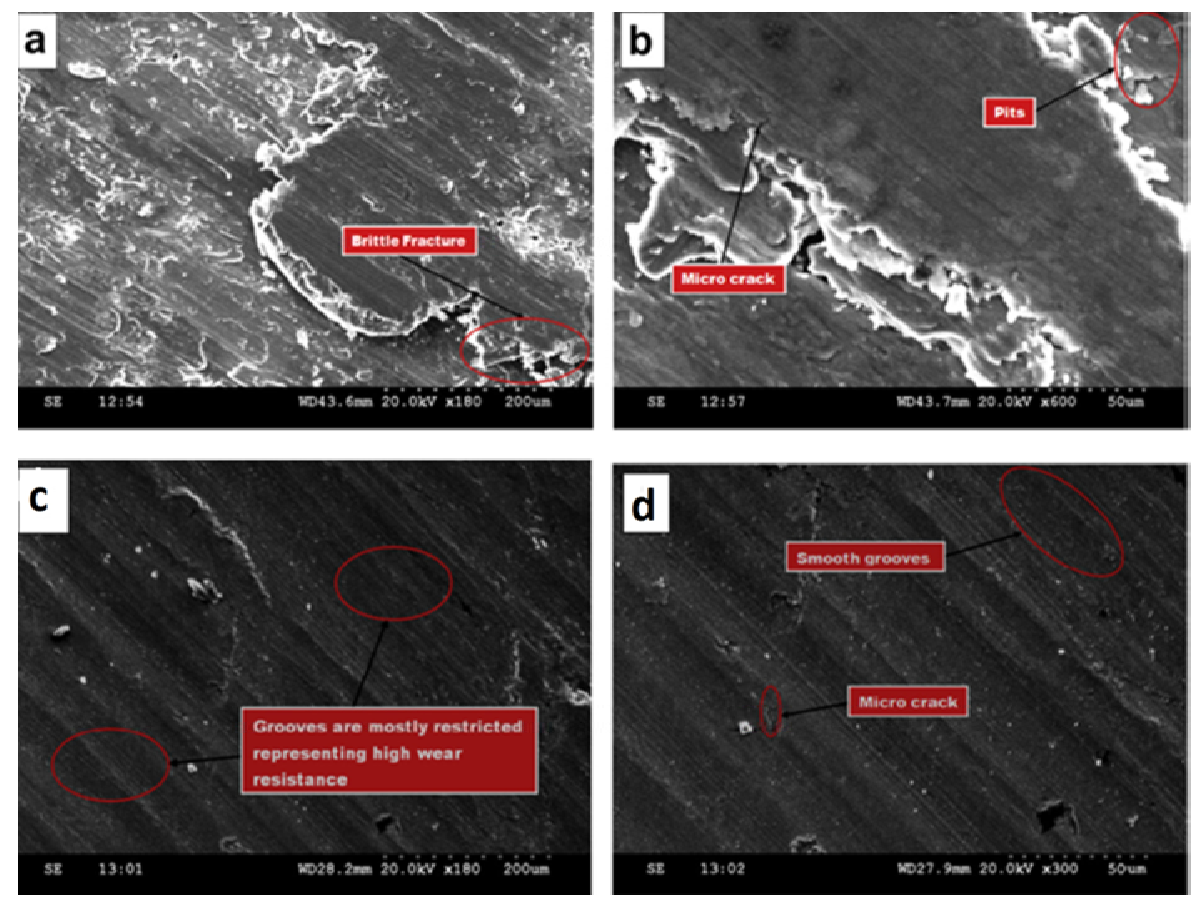

Fig. 5. SEM morphologies of the worn surface of nano-AI 2024 matrix (a) at low-magnification, (b) at highmagnification and $\mathrm{Al} / 5 \% \mathrm{SiC} / 10 \% \mathrm{Gr}$ hybrid nano-composite (c) at low-magnification, (d) at high-magnification on applied load of $20 \mathrm{~N}$, adapted from [35].

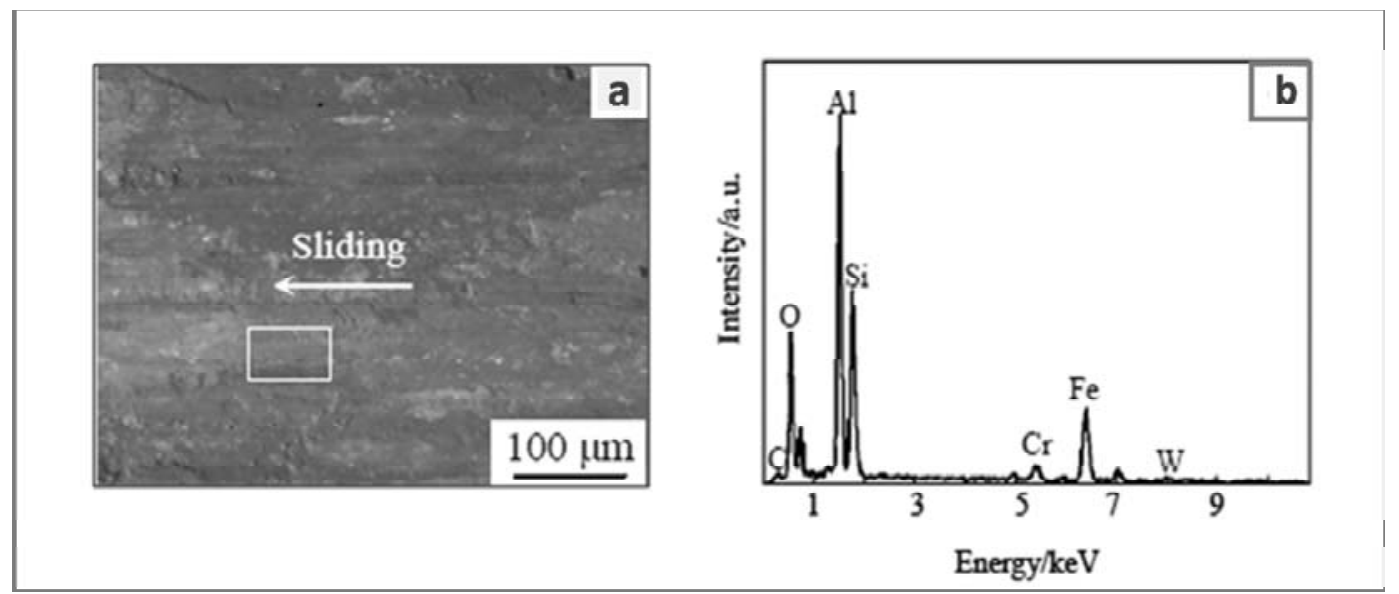

Fig. 6. (a) Optical micrographs of worn surface and (b) EDS analysis of the tribo-layer on the top surface (the area of rectangle in 6.a) of $\mathrm{Al} / \mathrm{SiC} / \mathrm{Gr}(20 \mu \mathrm{m})$ hybrid composite, adapted from [36].

composite possessed lower tensile properties as compared to base alloy, this lower strength was due to presence of $\mathrm{SiC}$ and $\mathrm{Al}_{2} \mathrm{O}_{3}$ particles which make the matrix brittle. S. Mahadevi and F. Akhlaghi [39] prepared a hybrid composite Al 6061/SiC/Gr via a new method which was combination of stir casting and powder metallurgy, named as in situ powder metallurgy(IPM). The hybrid composite reinforced with $\mathrm{Gr}$ and $\mathrm{SiC}$ decreased the compressibility and improved the hardness of hybrid composite. The hardness was decreased with reinforcement of $\mathrm{Gr}$ while increase with further reinforcing $\mathrm{SiC}$ as shown in Fig. 7. Through the microstructure analysis it was concluded that IPM method successfully distributed the SiC and graphite uniformly.

S. Prasat and R. Subramanian [40] synthesis AlSi10Mg/FlyAsh/Graphite hybrid composite using stir casting reinforcing low cost material fly ash and graphite. The tensile and hardness properties of hybrid composite were superior to AISi $10 \mathrm{Mg}$ alloy and AISi10Mg-Gr composite. This improvement in mechanical properties was due to presence of the hard and brittle fly ash particles which restrict the initiation of plastic deformation. Also a decline trend of density was shown with an increase in fly ash particle's content. N. Radhika et al. [41] synthesis 


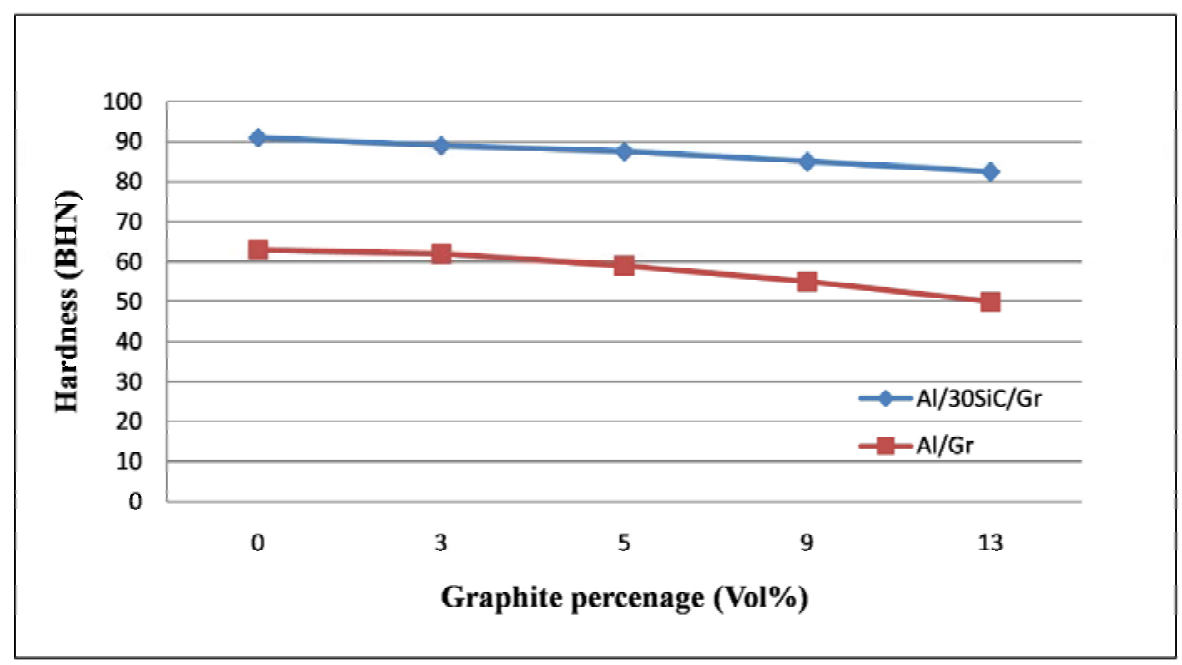

Fig. 7. Hardness values for $\mathrm{Al} / 30 \mathrm{SiC} / \mathrm{Gr}$ and $\mathrm{Al} / \mathrm{Gr}$ composites with different volume percent of graphite particles, adapted from [39].

AlSi10Mg/ $/ \mathrm{Al}_{2} \mathrm{O}_{3} / \mathrm{Gr}$ hybrid composite using stir casting by varying content of $\mathrm{Al}_{2} \mathrm{O}_{3}(3,6,9 \mathrm{wt} . \%)$ while keeping graphite content at 3 wt. $\%$. the experimentally investigation revealed that tensile strength and hardness of hybrid composite were improved with increase in wt $\%$ of $\mathrm{Al}_{2} \mathrm{O}_{3}$. M.V Krishna and A.M Xavior [42] investigated the mechanical properties of Al6061/SiC composites and Al6061/SiC/Gr hybrid composites prepared through stir casting technique. Al6061/SiC composites demonstrated the increase in density and tensile strength with increase in content of $\mathrm{SiC}$ at the expense of the hardness. Further hybrid composite reinforced with graphite showed increased tensile strength and reduced density with increase in graphite content. Microstructure analysis showed uniform distribution of $\mathrm{SiC}$ and Graphite in Al6061 matrix.

\section{TRIBOLOGICAL PROPERTIES OF HYBRID COMPOSITE}

According to G.W. Stachowiak and A.W. Batchelor [84] "Tribology is a field of science that applies an operational analysis to problems of great economic significance, such as reliability, maintenance and wear of technical equipment ranging from household appliances to spacecraft". Adalet Zen [46] investigated the influence of graphite on tribological properties of $\mathrm{Al} / \mathrm{SiC} / \mathrm{Gr}$ hybrid composite fabricated by powder metallurgy. The results revealed that addition of graphite in $\mathrm{Al} / \mathrm{Sic}$ composite reduced wear rate and improved lubrication properties. The percentage of graphite was varied from (0 to $8 \mathrm{wt} . \%$ ). The best wear resistance was exhibited on $6 \mathrm{wt} . \%$ of graphite addition. Further increasing graphite per- centage to $8 \%$ reduced the wear resistance. Abrasive wear was dominating wear mechanism for all $\mathrm{Al} / \mathrm{SiC} / \mathrm{Gr}$ hybrid composite specimens. P. Ravindran et al. [43] examined the tribological properties of graphite reinforced Al2024/SiC/Gr hybrid composite processed by powder metallurgy route. Graphite was added as solid lubricant in different contents $(0,5,10 \mathrm{wt} . \%)$. Due to self lubricating effect of graphite, wear rate and coefficient of friction of Al2024/SiC/Gr hybrid composites were decreased upto $5 \mathrm{wt} \%$ graphite reinforcement. Further increasing graphite content in hybrid composite resulted in increase the wear rate and coefficient of friction due to softness of graphite. So embedding graphite as secondary reinforcement in aluminum, effectively improves tribological properties. P. Ravindran et al. [35] also characterized the tribological behavior of Al2025/SiC/Gr hybrid composites reinforced with nano sized SiC (100 nm) and Graphite $(500 \mathrm{~nm})$. Hybrid nano composites were fabricated by powder metallurgy. The results showed that due to formation of tribo-layer of graphite during sliding at contact surface of $\mathrm{Al} / \mathrm{SiC} / \mathrm{Gr}$ hybrid composite with 10 wt.\% graphite, exhibited the lowest of wear loss. Wear loss was decreased with increase in graphite content. The overall results exhibited $\mathrm{SiC}$ and Graphite reinforced hybrid Aluminum nano composite as marvelous materials where high strength and wear resistance were key factors.

However there are some drawbacks of powder metallurgy such as need long time to achieve uniform distribution of particles during pressing and high fabrication costs. To overcome these limitations $S$. Mahdavi and F. Akhlaghi [10] studied new economi- 
cal method named as in situ powder metallurgy(IPM) combining two synthesizing techniques stir casting and powder metallurgy. Reinforcing $\mathrm{SiC}$ and Graphite showed improved wear rate and volume loss. At constant 9 vol.\% graphite and varying SiC from 0 to 40 vol. \%, after a distance of $1000 \mathrm{~m}$, the reduction in volume loss and wear rate was about $88 \%$, however further increase in $\mathrm{SiC}$ content to 40 vol. \%, increased the wear rate and volume loss by $76 \%$.In another excellent research by S. Mahdavi and F. Akhlaghi [39] revealed that Al6061/30SiC/Gr hybrid composite exhibited superior tribological properties than Al6061/Gr composite with identical graphite contents. The results revealed that wear rate and volume loss was increased with increase in content of graphite reinforcement in Al6061/Gr composites while there was decline trend of volume loss and wear rate with increase in graphite content in Al6061/ $\mathrm{SiC} / \mathrm{Gr}$ hybrid composite. The maximum wear resistance was obtained at Al6061/20SiC/9 Gr hybrid composite which exhibited $1 / 15^{\text {th }}$ and $1 / 30^{\text {th }}$ wear rate of Al 6061 alloy and Al6061/9Gr composite sample respectively. A. Baradeswaran et al. [56] examined the influence of graphite addition on tribological properties of $\mathrm{Al} 7075 / \mathrm{Al}_{2} \mathrm{O}_{3} / \mathrm{Gr}$ hybrid composite fabricated by liquid metallurgy route. It was experimentally found that hybrid composite incorporated with solid lubricating graphite material exhibited the superior wear resistance due to formation of this layer of graphite on the tribo surface. The dominating wear mechanism was abrasion and delamination. P. Ravindran et al. [43] investigated that incorporating $\mathrm{SiC}$ in aluminum 2024 alloy resulted increase in wear resistance which was further increased due to formation of graphite rich tribo-layer between sliding counterparts, when graphite was embedded as secondary reinforcement fabricating a $\mathrm{Al} / \mathrm{SiC} / \mathrm{Gr}$ hybrid composite. From micro structural analysis it was found that main wear mechanism was delimitation and oxidative for hybrid composite with $20 \mathrm{wt} \%$ $\mathrm{SiC}$ and $5 \mathrm{wt} \%$ Graphite. F.Akhlaghi et al. [85] fabricated Al2024-Gr composite via in situ powder metallurgy to investigate influence of graphite on bending strength, hardness and wear behavior in dry and oil impregnated sliding. From the experimental investigation it was found that the value of hardness and bending strength was decreased with varying graphite percentage from 0 to 20 wt.\%. Graphite percentage did not affect coefficient of friction of hybrid composite during oil impregnated sliding while during dry sliding wear rate of hybrid composite was decreased with reinforcing graphite up to $5 \mathrm{wt} . \%$. After 10 wt.\% graphite addition wear rate was in- creased due to adverse effect in formation of cracks resulting enhancement of delamination.

N.Radhika et al. [41] stated that aluminum based hybrid MMC's with SiC and Graphite as reinforcement exhibited significantly improved mechanical and tribological properties. The wear rate and coefficient of friction of stir casted Al/Sic/Gr hybrid composite was deceased with increase in sliding speed while increased with increase in applied load. From the microstructural examination of worn out specimen it was analyzed that damage was more in base alloys as compared to HMMC's, also depth and number of grooves was increased with increasing sliding speed and load. A.M. Hassan et al. [78] experimentally investigate the effect of Graphite and $\mathrm{SiC}$ reinforcement on hardness and surface roughness of compo cast Al- $4 \mathrm{wt} \% \mathrm{Mg}-\mathrm{Gr}$ composite, Al4wt.\%Mg-SiC composite and Al-4wt.\%Mg-Gr-SiC hybrid composites. They observed that Rockwell hardness of Al-4wt.\%Mg alloy was reduced with increase in graphite content and this reduction seems to be related to poor wettablity of graphite, its structure and lubricating behavior. However hardness was increased with increase in SiC content. Surface roughness of hybrid was improved with graphite addition, while reduced with $\mathrm{SiC}$ embedment. This improvement by $\mathrm{Gr}$ was due to its structure, lubricating action and it also act as chip breaker. Z.M Juan et al. [47] improved the wear resistance of AZ91D-Ce/9wt. $\% \mathrm{Al}_{2} \mathrm{O}_{3} / 15 \mathrm{wt} . \%$ graphite hybrid composite by addition of $\mathrm{Ce}$ (in weight \% of $0,0.4,0.8$, and 1 ) in form of cerium rich mg alloy. The hybrid composites were fabricated using squeeze infiltration technique. They found that $\mathrm{Ce}$ was enriched around the boundaries of $\mathrm{Gr}$ and formed $\mathrm{Al}_{3}$ Ce phase with Aluminum. Due to formation of this $\mathrm{Al}_{3} \mathrm{Ce}$ phase wear resistance was improved even at high load and best was on $1 \% \mathrm{Ce}$ addition. Abrasive and oxidation were dominating wear mechanism at low loads which were further changed into delamination at high loads. S.M. Shirazi et al. [11] experimentally assessed the effect of micron-sized graphite content on the hardness, corrosive and dry sliding wear behavior of Al6061/2SiC/Gr hybrid nano-composites. This hybrid composite were fabricated via powder metallurgy route. Their findings concluded that hardness was decreased with increase in Gr content. Al6061/2SiC/Gr hybrid composite possessed lower wear rate and coefficient of friction to that of Al6061/ 2SiC composites. However both friction coefficient and wear rate increased when graphite content was further increased from 2 vol.\% due to presence of brittle graphite particles. From the morphology of 


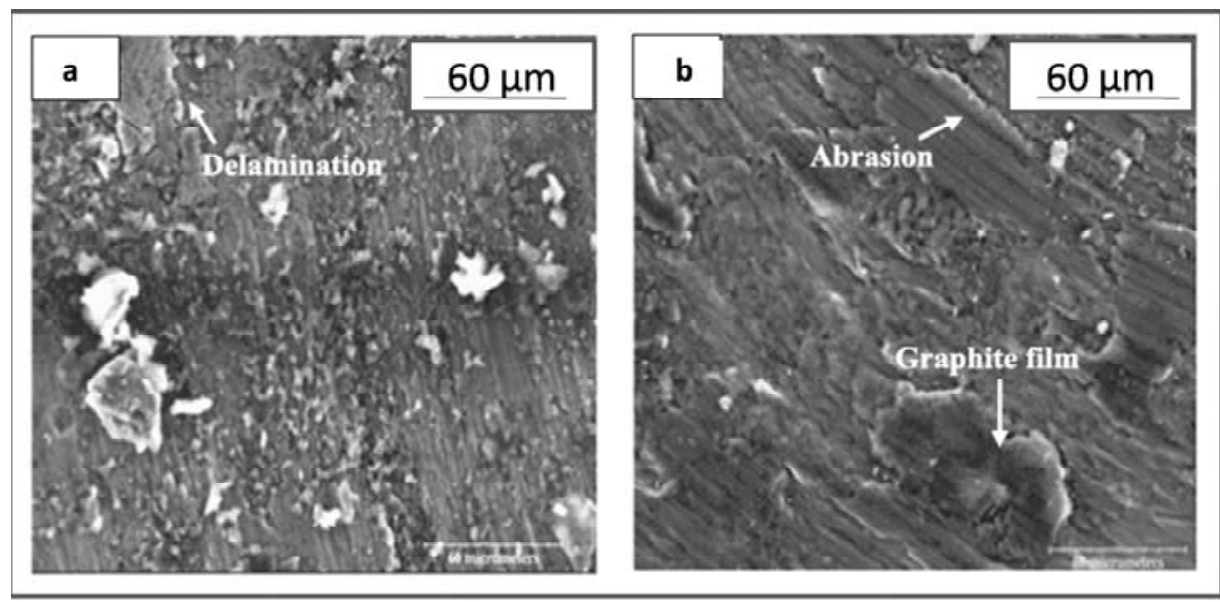

Fig. 8. SEM micrographs of the worn surfaces of a) $\mathrm{Al} / 2 \mathrm{SiC}$ and b) $\mathrm{Al} / 2 \mathrm{SiC} / 5 \mathrm{Gr}$ nano-composites adapted from [11].

worn surfaces it was revealed that dominating wear mechanism for Al6061/2SiC composites was delamination due to slight plastic deformation and presence of fine grooves; while continuous grooves on the surface of Al6061/2SiC /Gr hybrid composites suggested abrasion as dominanting wear phenomena as illustrated in Fig. 8.

N.C Kaushik and R.N. Rao [12] experimentally compared the tribological behavior of Al6082/SiC/ Gr hybrid composites with unreinforced Al6082 alloy and Al6082/SiC composites at load 5-15 N, 50$75 \mathrm{~m}$ sliding distance and $200 \mu \mathrm{m}$ grit size. They found that in both As cast and T6 heat treated Al/ $\mathrm{SiC} / \mathrm{Gr}$ hybrid composites wear resistance was superior to that of $\mathrm{Al} / \mathrm{SiC}$ composites, due to formation of tribo-layer of solid lubricant graphite. The SEM micrographs revealed that graphite reinforced hybrid Aluminum composites showed shallower grooves than base alloy and composites without graphite. S.V. Prasat and R. Subramanian [40] demonstrated that reduction in wear rate and coefficient of friction was observed with embedment of fly ash and graphite reinforcements in AISi10Mg alloy via stir casting method. The improved tribological properties of AlSi10Mg/ fly ash /Gr hybrid composite were due to load bearing ability of brittle fly ash particle and tribo-layer formed by solid lubricant graphite. SEM micrographs revealed the severe abrasive wear for AISi10Mg alloy showing coarse and deep grooves while abrasive wear for $\mathrm{Al} / 3 \mathrm{Gr}$ composite and mild abrasive wear for Al/9 fly ash /3Gr hybrid composite showing fine grooves. D.S.R. Smart et al. [34] assessed the influence of graphite and CNT on the mechanical, tribological and corrosion behavior of Al5083/Gr composite and Al5083/Gr/CNT hybrid composites. Experimentally it was revealed that reinforcing graphite in Al5083 alloy increased the wear resistance and corrosion resistance of composite while decreasing tensile strength (T.S); however this limitation of decrease in T.S was overcome by reinforcing hard CNT particles, having load bearing capacity in Al5083/Gr composite. This Al/ $\mathrm{Gr} / \mathrm{CNT}$ hybrid composite with $6 \mathrm{wt} \%$ graphite reinforcement exhibited superior wear resistance, T.S, hardness and corrosion resistance. T.Thirumalai et al. [86] synthesized and characterized the stir cast hybrid aluminum composite reinforced with $\mathrm{B}_{4} \mathrm{C}$ and $\mathrm{Gr}$. Their findings concluded that with increase in $\mathrm{B}_{4} \mathrm{C}$ content $(3,6,9,12 \%)$ while keeping graphite content constant, increases the hardness and wear resistance. Optimal content of $\mathrm{B}_{4} \mathrm{C}$ was shown $12 \%$ for loads varying from 10 to $40 \mathrm{~N}$, sliding distance of $500 \mathrm{~m}$ to $1000 \mathrm{~m}$ with a constant sliding speed of $1 \mathrm{~m} / \mathrm{s}$. A. Baradeswaran and A.E Perumal [87] demonstrated the effect of graphite on the tribological behavior of Al 7075-Gr composites, where graphite of size $16-20 \mu \mathrm{m}$ was reinforced at $5,10,15$, and 20 wt.\%. The results observed from the study indicated that wear rate and coefficient of friction were decreased with addition of $\mathrm{Gr}$ content upto $5 \mathrm{wt} . \%$ and this improvement was due to formation of graphitic film. However, further increasing Gr content results in increase of wear rate and COF; this seems to be related to graphite accumulation resulting squeeze out of the graphite from contact area. SEM micrograph of Al7075 alloy revealed severe patches and grooves which were further converted to mild patches and smaller grooves with 5 wt.\% graphite reinforcement to Al7075 alloy.

K.S Prakash et al. [48] synthesized Mg-SiC composites, Mg-Gr composites, and Mg-SiC-Gr hybrid composites via powder metallurgy and ex- 
amined the influence of reinforcements, applied load, sliding distance and sliding speed on wear loss and coefficient of friction of these composites. The results revealed that $\mathrm{SiC}$ reinforcement in magnesium, increased the micro hardness, COF, density and wear resistance of composite. So self lubricating solid lubricant graphite was further added for lowering COF and increasing wear resistance by forming a tribo-layer between sliding counterparts. Mg-SiCGr hybrid composites showed superior mechanical and tribological properties. However further increasing in graphite content reduced micro hardness and wear resistance due to increment of delamination and brittle fracture. B.M Girish et al. [75] experimentally examined that tribolgical behavior of AZ91 hybrid composites reinforced with $\mathrm{SiC}$ and $\mathrm{Gr}$, varied from 1 to $3 \%$ in $1 \%$ increments. The wear rate decreased with increase in content of $\mathrm{SiC}$ as well as Gr. This decrease in wear rate was due to load bearing capacity of composite. The increase in applied load results in transition of oxidation wear to abrasion and to delamination. $\mathrm{SiC}$ and $\mathrm{Gr}$ reinforcement in AZ91/SiC/Gr hybrid composite delayed this transition of oxidation to delamination wear. B.M Girish et al. [76] further optimized the wear rate of AZ91/SiC/Gr hybrid composite using Taguchi robust design technique. The parameters selected, which influence the stir casting process and wear rate were normal load in $\mathrm{N}(20,40,60)$, sliding speeds in $\mathrm{m} /$ $\mathrm{s}(1.045,1.51,2.09)$ and reinforcement in wt.\% of $\mathrm{SiC}$ and $\operatorname{Gr}(1,2,3)$. The lowest wear rate was revealed at $20 \mathrm{~N}$ load, $3 \mathrm{wt} . \%$ reinforcement and 1.047 $\mathrm{m} / \mathrm{s}$ sliding speed. The normal load (34.57\%) was the most significant factor followed by speed (20.75) and composition (11.70). S. Singh et al. [15] also optimized wear rate of $\mathrm{Al} 6082 / \mathrm{Al}_{2} \mathrm{O}_{3} / \mathrm{Gr} / \mathrm{B}_{4} \mathrm{C}$ hybrid composite prepared via stir casting using taguchi design technique. The influence of process and wear parameters involving normal load, track diameter and sliding distance was examined on wear behavior of hybrid composites. Normal load was found to be the most influencing parameter on wear rate. This was due to stick- slip phenomenon shown by increased frictional forces. S.Suresh and B.K. Sridhara [45] characterized friction coefficient of hybrid composite reinforced with $\mathrm{SiC}$ and Graphite varying at $2.6,5,7.5,10 \mathrm{wt} . \%$ of reinforcement, fabricated by stir casting. Analysis from the test which was according to design of experiments showed that sliding speed and load influence the friction coefficient while percentage reinforcement and sliding distance does not show significant impact. A. Sharma et al. [77] also optimized the wear rate and COF of stir cast Aluminum hybrid composite reinforced with varying Gr content $(0,3,6,9,12 \%)$ and Boron carbide content $(0,1,2,3,4 \%)$. The three influencing factors varied at three different levels were normal load, track diameter and sliding speed. Normal load was comes out the main significant parameter for both output responses wear rate and COF. N. Radikha and R. Subramaniam [14] also optimized tribological behavior (wear rate and COF) of AlSi $10 \mathrm{Mg} / \mathrm{Al}_{2} \mathrm{O}_{3} / \mathrm{Gr}$ hybrid composite fabricated via liquid metallurgy. L27 orthogonal array of taguchi was used for experiment design. The influencing control parameters were load, sliding speed and reinforcements. From the analysis of variance, applied load was found to be most significant factor followed by sliding speed and reinforceent. The combined interaction effect of applied load and sliding speed was the most significant on wear rate among other interaction with percentage contribution of $6.17 \%$. B.Stojanovic et al. [9] fabricated A356/SiC composites and A356/SiC/Gr hybrid composites using compos casting technique. The effect of different significant factors such as sliding speed, applied load, sliding distance and graphite reinforcement on the wear behavior of these composites and hybrid composites were analyzed. The A356/SiC/ Gr hybrid composite showed superior tribological properties than unreinforced alloys and composites. The results from factorial analysis revealed that effects of sliding distance, graphite content, sliding speed and applied load on wear rate were $11.25 \%$, $13.95 \%, 17.87 \%$ and $38.99 \%$ respectively. Also intersection effect of percentage of graphite and load is $3.29 \%$. For $A 356 / \mathrm{SiC} / \mathrm{Gr}$ hybrid composites the best wear resistance was achieved at $10 \% \mathrm{SiC}$ and $1 \%$ graphite reinforcement.

\section{CONCLUSION AND FUTURE SCOPE}

Self-lubricating hybrid composites have capability to achieve low friction coefficient and wear rate without providing any external supply of lubrication. This review presents an overview of research efforts focused on the tribology of aluminum and magnesium hybrid composites reinforced with hard ceramics materials like $\mathrm{SiC}, \mathrm{Al}_{2} \mathrm{O}_{3}, \mathrm{TiC}, \mathrm{B}_{4} \mathrm{C}$ as primary reinforcement and graphite as secondary reinforcement, which have tremendous applications as self lubricating materials. In this exhaustive review, considerable experimental results from the published researches have been discussed on the mechanical and tribological properties of aluminum and magnesium based self-lubricating HMMC's. The key ex- 
perimental-based conclusions obtained from the prior works carried out are briefly as follows:

i) The solid lubricant graphite has great potential as secondary reinforcement for superior mechanical and tribological properties of aluminum and magnesium based self-lubricating HMMC's. These materials are very important for green or environment friendly tribology, sustainability and energy efficiency, as oil and grease based lubricants releases harmful pollutants into environment.

ii) The graphite content within specific limits increases the wear resistance of hybrid composites at the expense of material's overall strength, which is overcome by impregnating hard ceramics like $\mathrm{SiC}, \mathrm{Al}_{2} \mathrm{O}_{3}, \mathrm{~B}_{4} \mathrm{C}$ in the hybrid Composites.

iii) When normal load increases, there is increase in real contact area which leads to increase in wear loss and coefficient of friction of composites. At low normal loads abrasive wear is the dominating wear mechanism. The transition point where abrasion and oxidation is further converted into delamination is exhibited at higher loads for graphite reinforced HMMC's as compared to unreinforced alloys.

iv) The literature review demonstrated that stir casting ( $45 \%)$ has been reported as the most contributing fabrication technique followed by powder metallurgy $(27 \%)$ for graphite impregnated self lubricating hybrid composites of $\mathrm{Al}$ and $\mathrm{Mg}$.

v) The combination of silicon carbide and graphite $(63 \%)$ is the most reported hybrid reinforcement followed by alumina and graphite (16\%) for fabrication of Al and Mg based self lubricating HMMC's.

vi) The literature reported in this paper inferred that most of the research work in the field of self-lubricating hybrid composites by reinforcing solid lubricant graphite has been carried out on aluminum alloys; however researchers are also concentrating on another metallic and ceramics materials such as light weight magnesium, titanium, zinc, copper, nickel, bronze silver, silicon nitride, alumina.

vii) The literature from the survey reported in this paper has also revealed that most of researchers focused only on few mechanical properties such as tensile strength and hardness of self lubricating hybrid composites; while properties like toughness, flexural strength, fatigue strength, compressive strength and creep resistance are less researched. viii) Applied load, sliding speed and composition of hybrid reinforcement are significant parameters discussed for optimization of tribological properties such as wear rate and coefficient friction of self lubricating hybrid composites of aluminum and magnesium. The applied load was most significant parameter reported among them. ix) Moreover the factors, during the fabrication of hybrid composites (e.g. for stir casting factors like stirrer speed, pouring temperature, solidification time, pre-mould temperature) could also be further studied for optimization of wear behavior. In this background, the tremendous contribution of researches presented in this paper provides research gaps and directions for future research in the field of graphite reinforced light weight hybrid composite for application in aerospace and automobile industries.

\section{REFERENCES}

[1] F.C. Campbell, Lightweight materialsUnderstanding the basics (ASM international, Ohio, 2012).

[2] J. Singh and A. Chauhan // J. Mater. Res. Technol. 5 (2016)159.

[3] S. B. Prabu , L. Karunamoorthy, S. Kathiresan and B. Mohan // J. Mater. Process. Technol. 171 (2006) 268.

[4] B. V. Ramnath, C. Elanchezhian, RM. Annamalai, S.Aravind, T. S. Atreya, V. Vignesh and C.Subramanian // Rev. Adv. Mater. Sci. 38 (2014) 55.

[5] A. Dey and K. Murari Pandey // Rev. Adv. Mater. Sci. 42 (2014) 581.

[6] P.K. Rohatgi, In: Tribology for Scientists and Engineers: From Basics to Advanced Concepts, ed. by M.T.Khorshid, E. Omrani, M. R. Lovell and P. L.Menezes (Springer Science, London, 2013), p.233.

[7] W.A. Monteiro, S.J. Buso and L.V. da Silva, In: New Features on Magnesium Alloys, ed. by W.A. Monteiro (Intech, Rijeka, 2012).

[8] P. Ravindran, K. Manisekar, S. Vinoth Kumar and P. Rathika // Mater. Des. 39 (2013) 42.

[9] B. Stojanovic, M. Babic, S. Velickovic, J. Blagojevic and S. Velickovic // Tribol. Trans.59 (2016) 522.

[10] S. Mahdavi and F. Akhlaghi // J. Mater. Sci. 46 (2011)1502.

[11] S. M. Shirazi, F. Akhlaghi and D. Y. Li // Tribol. Int. 103 (2016) 620.

[12] N. C. Kaushik and R. N. Rao // Tribol. Int. 96 (2016) 184.

[13] J. S. S. Babu, K. P. Nair, G. Unnikrishnan, C. G. Kang and H. H. Kim // J. Compos. Mater. 44 (2010) 971.

[14] N. Radhika and R. Subramaniam // Ind. Lubr. Tribol. 65 (2013) 166.

[15] S. Singh, M. Garg and N. K. Batra //Tribol. Trans. 58 (2015) 758. 
[16] K. Kanthavel, K. R. Sumesh and P. Saravanakumar // Alexandria Eng. J. 55 (2016) 13.

[17] D. Nayakab, N. Rayab, R. Sahoob and M. Debataa // Tribol. Trans. 57 (2014) 908.

[18] I. Aatthisugan, A. R. Rose and D. S. Jebadurai // J. Magnes. Alloys 5 (2017) 20.

[19] T. Thirumalai, R. Subramanian, S. Kumaran, S. Dharmalingam and S. S. Ramakrishnan // J. Sci. Ind. Res. 73 (2014) 667.

[20] J. Lu, S. Yang, J. Wang and Q. Xue // Wear 249 (2001) 1070.

[21] J. K. Xiao, L. Zhang, K. C. Zhou and X. P. Wang // Tribol. Int. 57 (2013) 38.

[22] X. C. Ma, G. Q. He, D. H. He, C. S. Chen and Z. F. Hu // Wear 265 (2008) 1087.

[23] Y.W. Wu, K.Wu, K.K. Deng, K.B. Nie, X.J. Wang, X.S. Hu and M.Y. Zheng // Mater. Sci. Eng. A 527 (2010) 6816.

[24] A. Baradeswaran and A. Elaya Perumal // Tribol. Trans. 58 (2015) 1.

[25] O. Carvalho, M. Buciumeanu, S. Madeira, D. Soares, F. S. Silva and G. Mirandam // Tribol. Int. 90 (2015) 148.

[26] M. T. Khorshid, E. Omrani, P. L. Menezes and P. K. Rohatgi // Eng. Sci. Technol. an Int. J. 19 (2016) 463.

[27] A. D. Moghadam, E. Omrani, P. L. Menezes and P. K. Rohatgi // Compos. Part B 77 (2015) 402.

[28] P. L.Menezes, In: Tribology for Scientists and Engineers: From Basics to Advanced Concepts, ed. by C.J. Reeves, M. R. Lovell, and P.K. Rohatgi (Springer Science, London, 2013), p. 341.

[29] B. Bhushan // Diam. Relat. Mater. 8 (1999) 1985.

[30] D. Sheeja, B. K. Tay, S. M. Krishnan and L. N. Nung // Diam. Relat. Mater. 12 (2003) 1389.

[31] M. Sedlacek, B. Podgornik and J. Vizintin // Mater. Charact. 59 (2008) 151.

[32] K. Oguri and T. Arai // Surf. Coat. Technol. 47 (1991) 710.

[33] M. Kalin, I. Velkavrh, J. Vizintin and L. Ozbolt // Meccanica 43 (2008) 623.

[34] D. S. R. Smart, N. P. Johns and J. J. Smart // Mater. Sci. Forum 889 (2016) 56.

[35] P. Ravindran, K. Manisekar, P. Narayanasamy, N. Selvakumar and R. Narayanasamy // Mater. Des. 51 (2013) 448.
[36] L. Jinfeng, T. Shoufu and C. Guoqin // Rare Met. Mater. Eng. 38 (2009) 1894.

[37] A. Baradeswaran and A. Elaya Perumal // Compos. Part B Eng. 56 (2014) 464.

[38] D. Aruri, K. Adepu, K. Adepu and K. Bazavada // J. Mater. Res. Technol. 2 (2013) 362.

[39] S. Mahdavi and F. Akhlaghi // Tribol. Lett. 44 (2011) 1.

[40] S. V. Prasat and R. Subramanian // Ind. Lubr. Tribol. 65 (2013) 399.

[41] N. Radhika, R. Subramanian, S. Venkat Prasat and B. Anandavel // Ind. Lubr. Tribol. 64 (2012) 359.

[42] M. V. Krishna and A. M. Xavior // Procedia Eng. 97 (2014) 918.

[43] P. Ravindran, K. Manisekar, P. Rathika, and P. Narayanasamy // Mater. Des. 45 (2013) 561.

[44] F. Akhlaghi and A. Zare-Bidaki // Wear 266 (2009) 37.

[45] S. Suresha and B. K. Sridhara // Mater. Des. $31(2010) 1804$.

[46] A. Zeren // Ind. Lubr. Tribol. 67 (2015) 262.

[47] M. J. Zhang, X. H.Yang, Y. B.Liu, Z.Y.Cao, L.R.Cheng, and Y.L.Pei // Trans. Nonferrous Met. Soc. China (English Ed.) 20 (2010) 207.

[48] K. S. Prakash, P. Balasundar, S. Nagaraja and P. M. Gopal// J. Magnes. Alloys 4 (2016) 197.

[49] J. Xiao, W. Zhang, L. Liu, L. Zhang and C. Zhang // Wear 385 (2017) 61.

[50] K. P. Furlan, P. C. Goncalves, D.R. Consoni, M.V.G. Dias, G. A.Lima, J. D. B. Mello and

A. N. Klein // J. Mater. Eng. Perform. 26 (2017) 1135.

[51] S. Sarkar and P. K. Das // Rev.Adv. Mater. Sci. 37 (2014) 53.

[52] A.M.K. Esawi, K. Morsi, A. Sayed, M. Taher and S. Lanka // Compos. Sci. Technol. 70 (2010) 2237.

[53] W. Zhang, G.J. Ma and C.W.Wu // Rev. Adv. Mater. Sci. 36 (2014) 75.

[54] I.A Ovid'ko // Rev.Adv. Mater. Sci. 38 (2014) 190.

[55] V.G. Konakov, I.A. Ovid'ko, N.V. Borisova, E.N. Solovyeva, S.N. Golubev,O.Y. Kurapova, N.N. Novik and I.Y. Archakov // Rev. Adv. Mater. Sci. 39 (2014) 41.

[56] A. Baradeswaran and A. E. Perumal // Compos. Part B 56 (2014) 464.

[57] K. Soorya Prakash, P. Balasundar, S. Nagaraja, P. M. Gopal and V. Kavimani // J. Magnes. Alloys 4 (2016) 197. 
[58] P. Shanmughasundaram and R. Subramanian // Adv. Mater. Sci. Eng. (2013) Article ID 216536

[59] P. Ravindran, K. Manisekar, R. Narayanasamy and P. Narayanasamy // Ceram. Int. 39 (2013) 1169.

[60] M. L. T. Guo and C.Y. A. Tsao // Composites science and technology 60 (2000) 65

[61] P. Sharma, K. Paliwal, R. K. Garg, S. Sharma and D. Khanduja // J. Asian Ceram. Soc. 5 (2017) 42.

[62] C. J. Reeves, In: Tribology for Scientists and Engineers: From Basics to Advanced Concepts, ed. by P. L. Menezes, M. R. Lovell and and T.C. Jen (Springer Science, London, 2013), p. 447.

[63] H.D. Wang, In: Encyclopedia of Tribology, ed. by Q.J. Wang and Y.W. Chung (Springer Science, London, 2013), p. 1550.

[64] M.O. Bodunrin, K. K. Alanemea, L. H. Chown // J. Mater. Res. Technol. 4 (2015) 434.

[65] J. L. Li and D. S. Xiong // Wear 265 (2008) 533.

[66] J. Li and D. Xiong // Wear 266 (2009) 360.

[67] Y. Wu, B. Shen, L. Liu, and W. Hu // Wear 261 (2006) 201.

[68] T. Ram Prabhu, V. K. Varma and S. Vedantam // Wear 317 (2014) 201.

[69] K. Rajkumar and S. Aravindan // Tribol. Int. 44 (2011) 347.

[70] K. H. W. Seah // Mater. Des. 16 (1995) 271.

[71] S. Mitrovi, M. Babi, B.Stojanovi and N. Miloradovi In: 12th International Conference on Tribology - Serbiatrib'11 (Kragujevac, Serbia, 2011), p. 138.

[72] M. Babic, M. Slobodan, D. Dzunic, B. Jeremic and B. Ilija // Tribol. Lett. 37 (2010) 401.

[73] K. K. Alaneme and O. J. Ajayi // J. King Saud Univ. - Eng. Sci. 29 (2017) 172.

[74] B. M. Girish, B. M. Satish, S. Sarapure, D. R. Somashekar and Basawaraj // Tribol. Trans. 58 (2015) 481.
[75] B. Girish, B. Satish and S. Sarapure // Metall. Mater. Trans. A 47 (2016) 3193.

[76] A. Sharma, M. Garg and S. Singh // Ind. Lubr. Tribol. 67 (2015) 380.

[77] A. M. Hassan, G. M. Tashtoush and J. A. Al-Khalil // J. Compos. Mater. 41 ( 2006) 453.

[78] Y. Feng, J. Wang, M. Zhang and Y. Xu // J. Mater. Sci. 42 (2007) 9700.

[79] X. Wang, Z. Wen, B. Lin, J. Lin, X. Wu and X. Xu // J. Power Sources 184 (2008) 508.

[80] S. K. Maiti, S. Sarkar, M. F. Wani and P. K. Das // Metall. Mater. Trans. A 46 (2015) 5719.

[81] A. Gangopadhyay // Tribol. Trans. 34 (1991) 257.

[82] G. Cui, Q. Bi, M. Niu, J. Yang and W. Liu // Tribol. Int. 60 (2013) 25.

[83] S.Srivastava, S. Mohan, Y. Srivastava and A. J. Shukla // International Journal of Modern Engineering Research 2 (2012) 25.

[84] G.W. Stachowiak and A.W. Batchelor, Engineering Tribology (Elsevier Ltd,U.K 1993).

[85] F. Akhlaghi and Zare-Bidaki // Wear 266 (2009) 37.

[86] T. Thirumalai, R. Subramanian, S. Kumaran, S. Dharmalingam and S. S. Ramakrishnan // J. Sci. Ind. Res. 73 (2014) 667.

[87] A. Baradeswaran and A. E. Perumal // Compos. Part B56 (2014) 472.

[88] Y.W. Wu, K.Wu, K.K. Deng, K.B. Nie, X.J. Wang, X.S. Hu and M.Y. Zheng // Mater. Sci. Eng. A 527 (2010) 6816.

[89] F. Akhlaghi and S. Mahdavi // Adv. Mater. Res. 264-265 (2011) 1878.

[90] K.R .Gopi, K.N. Mohandas, H.N.Reddappa and M.R. Ramesh // Int. J. Eng. Adv. Technol. 2 (2013) 340.

[91] Z. Liu, G. Zu, H. Luo, Y. Liu and G. Yao // J. Mater. Sci. Technol. 26 (2010) 244.

[92] E. Gewfiel, M. A. H. El-Meniawi and Y. Fouad, In: International Conference on Engineering and Technology (ICET) (Cairo, Egypt, 2012), p. 1. 